\title{
基于功能离子液体促进的有机反应研究进展
}

\author{
徐慧婷 ${ }^{a}$ 张超怀 $b$ 陈 钢 ${ }^{b}$ 沈润溥*, $a$ 应安国*, $b$ \\ ( ${ }^{a}$ 绍兴文理学院化学化工学院 绍兴 312000) \\ $\left({ }^{b}\right.$ 台州学院医药化工学院 台州 318000)
}

\begin{abstract}
摘要 离子液体作为绿色溶剂和催化剂, 具有低挥发性, 优良的热稳定性、溶解性以及可回收等优点, 近年来广泛应用 于有机合成领域中，在提高反应速率，反应选择性等方面发挥了巨大作用. 综述了离子液体作为催化剂或溶剂在传统 有机合成反应中的最新研究成果，包括 Baylis-Hillman 反应、Michael 加成反应、氧化还原反应、Knoevenagel 缩合反应、 Aldol 缩合反应、Diels-Alder 反应、Heck 偶联反应、Suzuki 反应、不对称反应、Biginelli 反应、Mannich 反应、Hantzsch 多组分反应. 分析离子液体结构对反应催化性能的影响, 探讨其反应机理, 为开发新型结构可调性功能离子液体打下 基础.
\end{abstract}

关键词 功能离子液体; 有机合成; 构效关系; 绿色化学; 重复使用

\section{Application of Task-Specific Ionic liquids to Organic Synthesis}

\author{
$\mathrm{Xu}$, Huiting $^{a} \quad$ Zhang, Chaohuai $^{b} \quad$ Chen, Gang $^{b} \quad$ Shen, Runpu*,a $\quad$ Ying, Anguo ${ }^{*, b}$ \\ ( ${ }^{a}$ School of Chemistry and Chemical Engineering, Shaoxing University, Shaoxing 312000) \\ ( ${ }^{b}$ School of Pharmaceutical and Chemical Engineering, Taizhou University, Taizhou 318000)
}

\begin{abstract}
Ionic liquids (ILs) have been widely used as green reaction solvents and/or catalysts in organic synthesis due to their advantages, including low volatility, good thermal stability, good solubility and recyclability. ILs have played a significant role in accelerating reaction rate and selectivity. The recent advances in the application of ILs as catalysts or solvents are summarized in this review, such as in Baylis-Hillman reaction, Michael addition, oxidation and reduction reaction, Knoevenagel condensation, aldol reaction, Diels-Alder reaction, Heck coupling reaction, Suzuki reaction, asymmetric reaction, Biginelli reaction, Mannich reaction and Hantzsch reaction. Relationship between structure and catalytic efficiency of ILs as well as plausible mechanism has been discussed, which is beneficial to develop the novel task-specific ionic liquids with diverse functionalities.
\end{abstract}

Keywords task-specific ionic liquids; organic synthesis; relationship between structure and activity; green chemistry; recyclability

近几年, 离子液体(Ionic liquids, ILs) 作为绿色溶 剂 ${ }^{[1 ~ 3]}$ 和环境友好的催化剂 ${ }^{[4 \sim 6]}$ 广泛运用于有机化学和 生物化学领域 ${ }^{7 \sim 10]}$, 是新兴的研究热点之一. 离子液体 是指完全由有机阳离子和有机、无机阴离子所组成的一 种常温熔融盐, 也称为低温熔融盐. 有机阳离子主要分 为四类: 咪唑类、季铵类、吡啶类、季鏻类, 阴离子则 几乎涵盖了所有有机、无机阴离子, 代表性的有: $\mathrm{HSO}_{4}^{-} 、 \mathrm{NTf}_{2}^{-} 、 \mathrm{BF}_{4}^{-} 、 \mathrm{BF}_{6}^{-} 、 \mathrm{CF}_{3} \mathrm{COO}^{-[11,12]}$. 由于 $\mathrm{ILs}$ 具有优异的热稳定性, 良好的溶解性, 可忽略的蒸气压,
且可以根据实验具体要求调节酸度, 回收可循环使用等

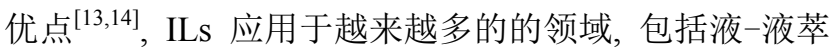
取 $^{[15]}$ 、湿法冶金 ${ }^{[16]}$ 、材料化学 ${ }^{[17]}$ 、电化学 ${ }^{[18 \sim 22]}$ 等. 此外, ILs 可以作为非均相催化剂, 相比传统均相催化剂昂贵 的价格，麻烦的后处理以及最终形成污染环境的金属配 合物, IL 不仅具有技术优势, 而且具有较强的经济竞争 力和环境竞争力, 符合绿色化学的道路.

结合本课题组在新型功能离子液体的设计、开发和 应用方面的成果 ${ }^{[23 ~ 25]}$, 本文对它们在传统有机合成单

*E-mail: yinganguo@163.com; srunpu@usx.edu.cn

Received April 26, 2016; revised May 28, 2016; published online June 8, 2016.

Project supported by the Natural Science Foundation of Zhejiang Province (No. LY15B060002), the National Natural Science Foundation of China (Nos. 21576176, 21106090), and the Science and Technology Innovation Undergraduate Program of Zhejiang Province (No. 2015R430015).

浙江省自然科学基金(No. LY15B060002)、国家自然科学基金(Nos. 21576176, 21106090)、浙江省大学生科技创新活动计划(No. 2015R430015)资助项 目. 
元中的应用作系统的归纳和总结, 主要包括加成反应、 氧化还原反应、缩合反应、偶联反应、不对称反应和多 组分反应. 然后对离子液体的结构与催化活性关系进行 分析, 指出目前存在的一些问题以及将来努力改进的方 向.

\section{1 加成反应}

\subsection{Baylis-Hillman 反应}

Baylis-Hillman (B-H)反应是一个形成 C-C 键的原 子经济反应 ${ }^{[26]}$, 在以往的 B-H 反应中, 常用的溶剂为乙 腈、四氢呋喃、二氯甲烷等, 随着对反应研究的深入, 化 学家们发现将离子液体作为反应溶剂应用于 $\mathrm{B}-\mathrm{H}$ 反应 中有利于加快反应速度和提高反应产率.

Zhao 研究小组 ${ }^{[27]}$ 合成了新型的聚乙二醇-400 离子 液体(IL-400，1)应用于 Morita-Baylis-Hillman 反应(Eq. 1). 研究发现, IL-400 复合溶剂体系有效地促进了各种 芳香醛及脂肪醛的 Morita-Baylis-Hillman 反应, 不仅反 应速度快，而且具有理想收率(63\% 93\%). 研究发现， IL-400 可以至少循环使用 5 次, 保持反应收率基本不变.<smiles>Cc1ccc(S(=O)(=O)[O-])cc1</smiles>

$$
\begin{aligned}
& \mathrm{R}^{1}-\mathrm{CHO}+\int^{\mathrm{R}^{2}} \underset{\mathrm{DABCO}, \text { r.t. }}{\stackrel{1, \mathrm{H}_{2} \mathrm{O}}{\longrightarrow}} \\
& \mathrm{R}^{1}=\text { Aryl, Alkyl } \\
& \mathrm{R}^{2}=\mathrm{CN}, \mathrm{ROOCH}_{3}
\end{aligned}
$$

Zhao 等 ${ }^{[28]}$ 还报道了以廉价的三丁基膦和各种卤代 烃为原料, 制备一系列季鏻离子液体 $\mathbf{2}$, 然后与水组成 复合体系, 用于催化 Morita-Baylis-Hillman 反应. 考察 发现, 在该复合溶剂体系中, 各种醛与丙烯腈、丙烯酸 甲酯及丙烯酰胺可以在室温下发生 Morita-BaylisHillman 反应, 反应速率快、收率高.

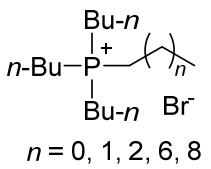

2a

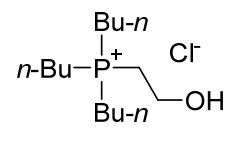

2b
$\mathrm{Zou}$ 等 ${ }^{[29]}$ 报道了质子离子液体 DABCO-AcOH- $\mathrm{H}_{2} \mathrm{O}$ 作为溶剂-催化剂体系参与的 Baylis-Hillman 反应(Eq. 2). $3 \sim 5$ equiv. 的水与有机盐形成水合物, 获得高熔点的 有机盐离子液体, 该溶剂-催化剂体系适用于芳香醛、脂 肪醛和丙烯酸酯、丙烯腈等受体. 反应粗品溶于甲苯后
易与离子液体分开, 少量残留在甲苯中的离子液体也可 以用水萃取后重复使用. DABCO-AcOH- $\mathrm{H}_{2} \mathrm{O}$ 溶剂-催化 系统可以重复使用 5 次，没有明显的活性损失.

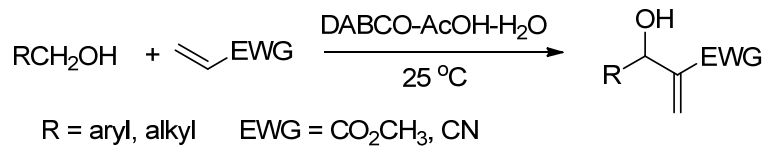

Lenardao 等 $^{[30]}$ 报道了含硒酸性离子液体 [pbeSe]$\mathrm{BF}_{4}(3)$ 催化的酫和缺电子烯烃之间的 Baylis-Hillman 反 应(Eq. 3). 研究表明, 催化剂 3 可使反应在室温下进行, 有较高的收率，且有利于减少反应时间. 他们认为反应 过程中 $[\mathrm{pbeSe}] \mathrm{BF}_{4}$ 形成的稳定的硒盐加速了反应速率, 反应机理如下 Scheme 1, 首先 1,4-二氮杂二环[2.2.2]辛 烷(DABCO)攻击贫电子烯烃, 得到烯醇盐, 紧接着羟醛 缩合得到两性中间体 $\mathbf{a}$, 最后分子内质子转移脱去催化 剂得到产物.
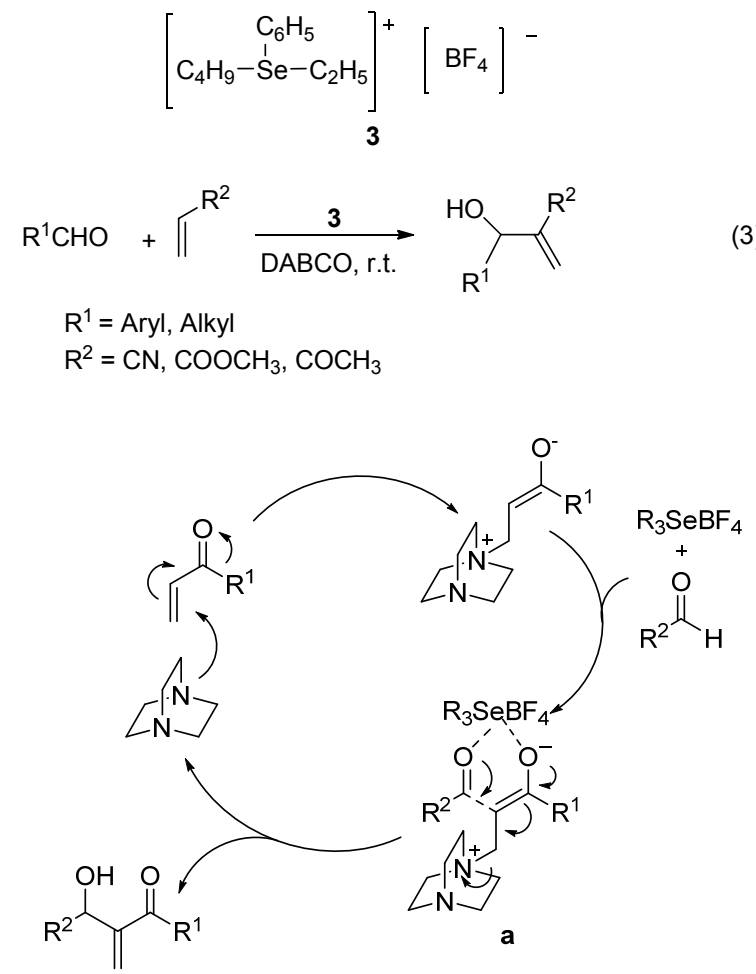

图式 1 [pbeSe]BF 4 催化的 Baylis-Hillman 反应机理 Scheme 1 Plausible mechanism of Baylis-Hillman reaction catalyzed by $[\mathrm{pbeSe}] \mathrm{BF}_{4}$

\subsection{Michael 加成}

Michael 加成反应在有机合成中是一种高效、原子 经济性的形成 $\mathrm{C}-\mathrm{C}$ 键的重要方法，是活泼亚甲基化物 烷基化和增长碳链的重要手段之一.

Laitonjam 等 ${ }^{[31]}$ 报道了 1,4 -二氮杂双环 [2.2.2]辛烷 (DABCO) 系列离子液体 4 催化活性亚甲基化合物与 $\alpha, \beta$ 不饱和酯、腈的 Michael 加成反应(Eq. 4). 该反应在室 
温下进行, 无需溶剂且收率高 $(85 \% \sim 100 \%)$, 反应结束 后, 产品以纯的固体或液体形式分离出来. 研究发现, 该催化体系活性受离子液体烷基支链长度和阴离子种 类影响, 其中 [C4DABCO]OH 为催化剂、用量为 $5 \mathrm{~mol} \%$ 时催化效果最好 $(97 \% \sim 100 \%)$, 可循环使用 6 次, 活性 基本保持不变.

$$
\sim_{n}^{+} \mathrm{A}^{-} \begin{aligned}
& n=4 \sim 9 \\
& \mathrm{~A}=\mathrm{Cl}, \mathrm{OH}
\end{aligned}
$$

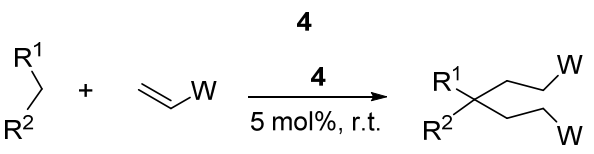

$$
\begin{aligned}
& \mathrm{R}^{1}, \mathrm{R}^{2}=\mathrm{COOMe}, \mathrm{COOEt}, \mathrm{CN}, \mathrm{COMe} \text {, etc. } \\
& \mathrm{W}=\mathrm{COOMe}, \text { COOEt, } \mathrm{CN} \text {. }
\end{aligned}
$$

Belfaitah 等 ${ }^{[32]}$ 合成了离子液体 1-丁基-3-甲基咪唑 四氟嗍酸盐([bmim $\left.] \mathrm{BF}_{4}, \mathbf{5}\right)$ 用于催化分子内氮杂 Michael 加成反应(Eq. 5). 该反应的产品经乙醚萃取后易与离子 液体分离, 且底物苯环上的电子和立体效应对反应产物 基本没有影响. 5 在该反应中作为溶剂和催化剂, 可以 循环利用 3 次, 活性稍微下降, 重复使用三次后, 活性 下降 $11 \% \sim 19 \%$.<smiles>CCCCn1cc[n+](C)c1</smiles><smiles>Nc1ccccc1C(=O)/C=C/c1ccccc1</smiles>

我们课题组 ${ }^{[33,34]}$ 以 DABCO 和 3-氯-1, 2-丙二醇为原 料合成了一系列新的碱性离子液体 $\mathbf{6}$, 用于催化 $\alpha, \beta$-不 饱和酰胺和胺类化合物的无溶剂氮杂 Michael 加成反应 (Eq. 6), 研究发现, 离子液体[DABCO-PDO][OAc]催化 效果最好, 相较其它催化剂 $\left(\mathrm{X}^{-}=\mathrm{BF}_{4}^{-}, \mathrm{BF}_{6}^{-}, \mathrm{CF}_{3} \mathrm{SO}_{3}\right)$ 反应时间短, 收率更高(高出 $4 \% \sim 9 \%$ ). 催化剂回收后 可重复使用 8 次, 保持较高的催化活性. 反应机理如 Scheme 2 所示: 离子液体 $\mathrm{N}$ 原子上的孤对电子带走胺基 上的氢原子形成活性氮负离子, 氮负离子进攻 $\mathrm{C}-\mathrm{C}$ 双 键的端基碳; 另一方面, 离子液体的羟基与羰基氧形成 氢键, 得到氧负离子, 烯醇异构得到酩. 我们利用红外、 碳谱和化学计算(图 1)多方面证实氢键的存在.

\section{2 氧化还原反应}

\section{1 氧化反应}

Wang 等 ${ }^{[35]}$ 研究了以离子液体为溶剂的级联 oxa-

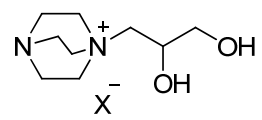

$\mathrm{X}=\mathrm{OAc}, \mathrm{BF}_{4}, \mathrm{PF}_{6}, \mathrm{CF}_{3} \mathrm{SO}_{3}$.

6

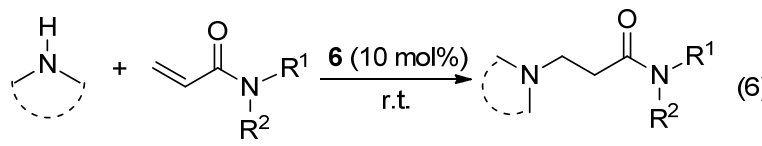

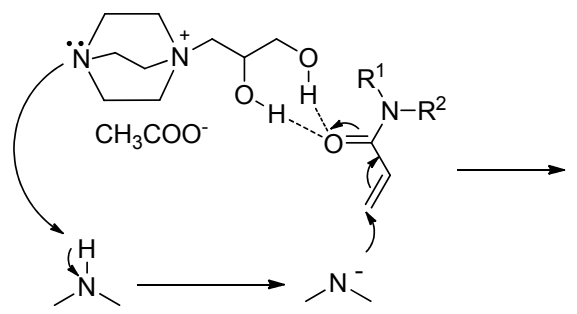<smiles>[R]N([R])C(=O)CCN1CCC1</smiles>

图式 2 [DABCO-PDO][X]催化的 Michael 加成反应机理 Scheme 2 Mechanism for Michael addition reaction catalyzed by [DABCO-PDO][X]

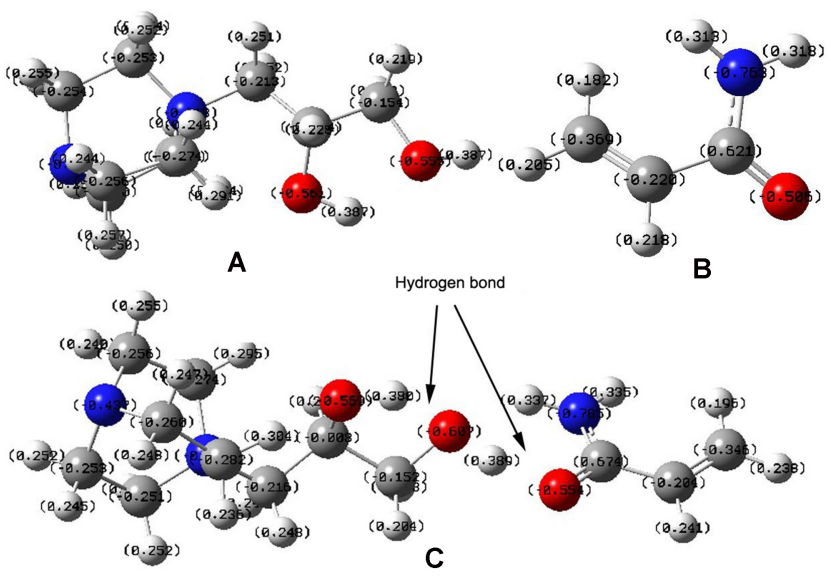

图 1 DABCO-PDO (A), 丙烯酰胺 (B) 以及丙烯酰胺与 [DABCO-PDO][OAc]混合物 $(\mathrm{C})$ 的电荷图

Figure 1 Charges of DABCO-PDO (A), acrylamide (B) and acrylamide-[DABCO-PDO][OAc] mixture (C)

Michael 氧化反应(Eq. 7), 以 $\mathrm{CuI}$ 为催化剂, 查尔酮为底 物, 反应条件温和, 温度低, 产物收率高(可达 98\%). 研 究证明, 在分子溶剂中该反应所需时间长且温度高达 $130{ }^{\circ} \mathrm{C}$, 以 $[\mathrm{bmim}]^{+}$系列离子液体为反应溶剂大大降低 了反应时间和温度 $\left(50^{\circ} \mathrm{C}\right)$. 其中 $[\mathrm{bmim}]\left[\mathrm{NTf}_{2}\right]$ (7)效果最 好, 反应迅速，可以在较短的时间内得到高收率 $(0.5 \mathrm{~h}$, 收率 94\%). 


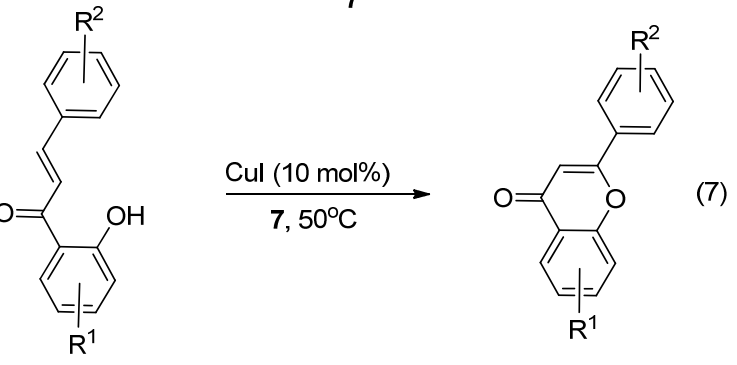

Vos 等 ${ }^{[36]}$ 研究了咪唑类离子液体与钯组合用于非活 化醇类的氧化反应 (Eq. 8). 研究发现, 离子液体 $[\mathrm{bmim}]\left[\mathrm{BF}_{4}\right](\mathbf{5})$ 和 $[\mathrm{emim}][\mathrm{TsO}](\mathbf{8})$ 效果较好, 当原料为 二癸醇时, 收率可达 $86 \%$. 反应终止后, 冷却至室温, 在该类离子液体中酮类产物具有清晰的相分离, 可以直 接倾析进行产物分离, 不需要有机溶剂萃取或蒸馏. 此 外, 离子液体还充当催化剂的固定化介质, 允许催化剂 循环使用，回收后选择性保持 $100 \%$, 收率没有大幅度 下降.

$$
\begin{aligned}
& \widehat{L}_{\mathrm{N}}^{\mathrm{N}_{\mathrm{N} O}^{+}-} \\
& 8
\end{aligned}
$$

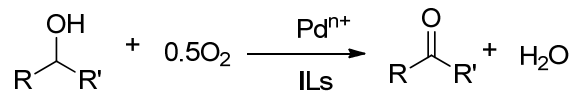

\section{2 还原反应}

苯偶酰可作为增味剂、杀虫剂、光敏胶和光固化涂 料的光固化剂及医药中间体 ${ }^{[37,38]}$. 传统的合成方法一般 需要铬试剂, 较高的反应温度, 且收率低.

Sahiner 等 ${ }^{[39]}$ 报道了鞣酸-铜离子液体 $[\mathrm{p}(\mathrm{TA})-\mathrm{Cu}]$ 催 化的 4-硝基苯酚(Eq. 9)、亚甲基蓝(MB，Eq. 10)和曙红 (Eq. 11)的还原反应. 该体系下 4-硝基苯酚的硝基还原 为胺基, $\mathrm{MB}$ 还原得到无色的 $\mathrm{LMB}$, 相较传统的嗍氢化 钠催化反应 ${ }^{[40]}, \mathrm{MB}$ 在 $\mathrm{P}(\mathrm{TA})-\mathrm{Cu}$ 离子液体中收率高, 反 应时间短. 曙红苯环上的羰基被还原, 在无催化剂的条 件下曙红还原速率很慢 ${ }^{[41]}$, 而该反应在 $\mathrm{P}(\mathrm{TA})-\mathrm{Cu}$ 离子 液体的作用下，10 $\mathrm{min}$ 内基本反应完全. 该研究中的还 原反应速率快且生成物无毒, 在处理有机硝基化合物和 染料污染方面具有巨大的实际应用潜力.<smiles>CC(Br)(Br)C(Br)=Cc1cc(N)ccc1O</smiles>
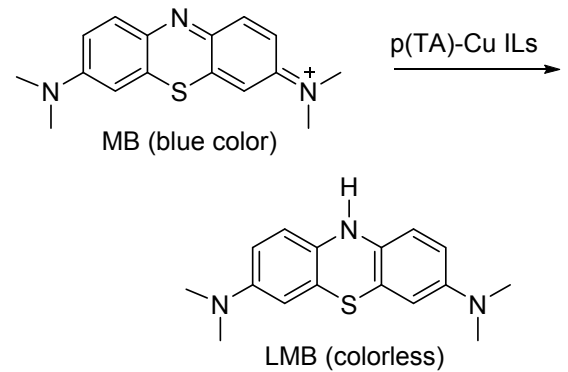

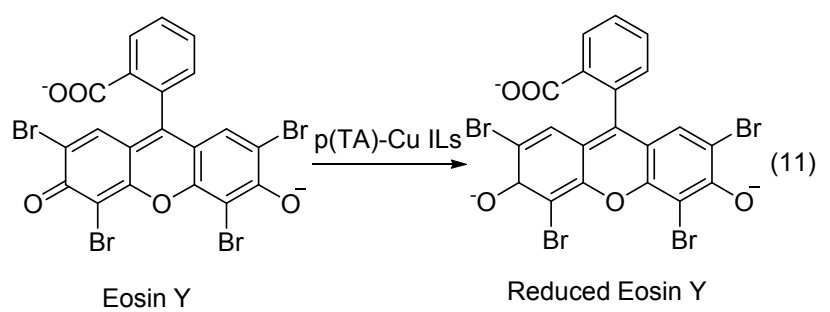

Singh 课题小组 ${ }^{[42]}$ 研究了 $\alpha, \alpha$-二苯基- $(L)$-脯氨修饰 的咪唑类离子液体 $\mathbf{9} \sim \mathbf{1 0}$ 和 $\mathrm{BH}_{3} \cdot \mathrm{SMe}_{2}$ 组合催化的苯乙 酮的不对称还原反应(Eq. 12). 研究发现, 10 的稳定性更 好, 10 的醚键相较 9 的酯键更加稳定, 并且具有良好的 收率(94\% 99\%)和光学纯度 $(e e$ 值, $81 \% \sim 88 \%$ ). 该反 应在 $70{ }^{\circ} \mathrm{C} 、 \mathrm{THF}$ 溶剂中进行，回流 $1 \mathrm{~h}$ ，反应完全，离 子液体回收后可至少重复使用 4 次，并且保持高收率 (91\% 99\%)和 $e e$ 值( $81 \% \sim 87 \%)$.
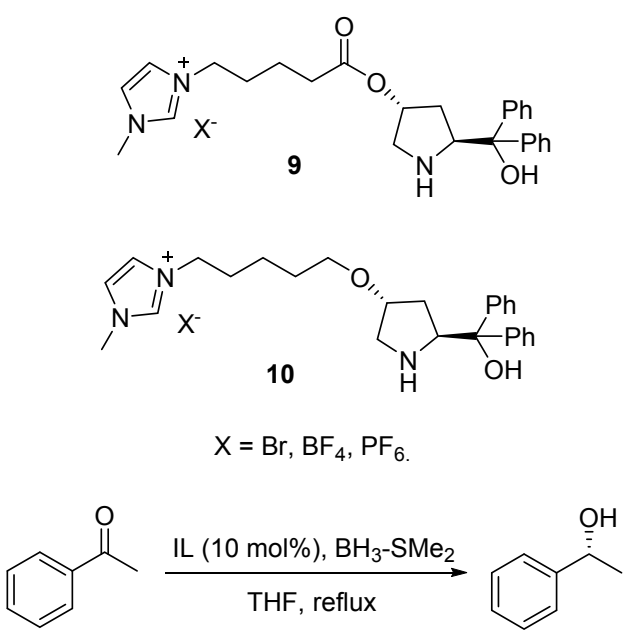

$\mathrm{Kim}$ 研究小组 ${ }^{[43]}$ 报道了离子液体 $\left[\mathrm{C}_{6}(\mathrm{mpy})_{2}\right]-$ $\left[\mathrm{NiCl}_{4}\right]^{2-}$ (11) 负载于 PVDF 纳米纤维得到复合材料 PVDF-IL 催化的芳香族硝基化合物的还原反应(Eq. 13). 离子液体中的镍、卤化物阴离子很好地分散在纳米纤维 中, 在 $\mathrm{NaBH}_{4} / \mathrm{H}_{2} \mathrm{O}$ 溶剂中有效地催化芳族硝基化合物 为苯胺. 研究发现, PVDF-IL 比 11 催化活性高, 室温反 应 $15 \mathrm{~min}$ 即得理想收率(76\% 90\%)且不影响芳环上的 各种官能团. 另外, PVDF-IL 制备简单、操作方便, 不需 要强酸性介质、压力设备, 成本低. 
<smiles>Cc1ccc[n+](CC(C)(C)C(C)(C)C(C)(C)C[n+]2cccc(C)c2)c1</smiles>

11

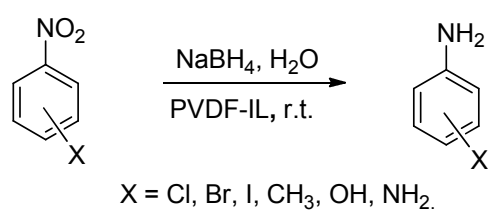

\section{3 缩合反应}

\subsection{Knoevenagel 缩合}

醛与活性亚甲基化合物之间的 Knoevenagel 缩合是 形成碳碳双键重要的有机反应之一 ${ }^{[44,45]}$. 缩合产物 $\alpha, \beta$ -

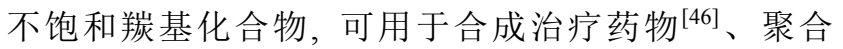
物 ${ }^{[47]}$ 、化妆品和香料 ${ }^{[48]}$ 的关键中间体.

$\mathrm{Tao}$ 等 ${ }^{[49]}$ 利用简单的中和反应合成了四丁基膦氨基 酸盐离子液体([ $\left.\left.\mathrm{P}_{4444}\right][\mathrm{AA}], 12\right)$, 在无溶剂条件下催化各 种芳香醛和活性亚甲基化合物的 Knoevenagel 缩合反应 (Eq. 14), 具有良好的收率 $(85 \% \sim 93 \%)$. 分析发现， $\left[\mathrm{P}_{4444}\right][\mathrm{AA}]$ 的催化活性受 $\mathrm{pH}$ 值和阴离子的影响, 强碱 性有助于提高反应收率. 当阴离子为脯氨酸时, 催化效 果最好, $40{ }^{\circ} \mathrm{C}$ 下反应 $20 \mathrm{~min}$, 收率达 $93 \%$. 相较于传统 的 Knoevenagel 缩合反应, 该反应无需溶剂、反应时间 短、温度低、催化剂用量少. $\left[\mathrm{P}_{4444}\right][\mathrm{Pro}]$ 可以至少重复使 用 6 次, 保持活性基本不变. 可能的反应机理如 Scheme 3 所示: 催化剂 $\left[\mathrm{P}_{4444}\right][\mathrm{Pro}]$ 上的仲胺通过氢键活化芳香 醛的羰基, 氧负离子带走氰化物亚甲基上的氢, 形成的 碳负离子作为亲核试剂进攻羰基碳, 电荷顺势转移到羰 基氧上，最后脱水形成双键.

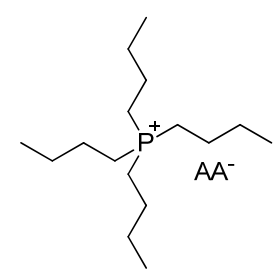

$[\mathrm{AA}]=$ Pro, Val, Gly, Ser.

12

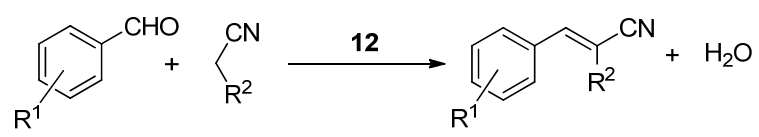

Bahrami 等 ${ }^{[50]}$ 报道了 Brønsted 酸性离子液体乙烷1,2-二铵硫酸氢盐(EDAHS, 13)在十二烷基硫酸钠(SDS) 的水溶液中催化芳香醛与丙二腈、巴比妥酸及 $1,3-$ 二甲 基巴比妥酸的 Knoevenagel 缩合反应(Eq. 15). 他们认为

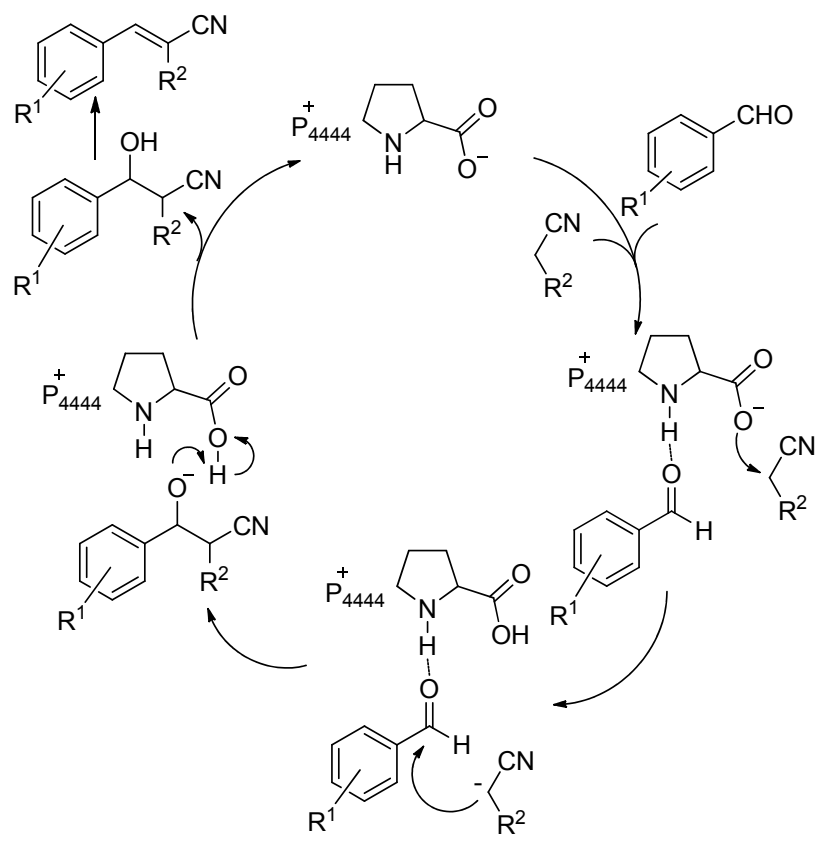

图式 3 EDAHS 参与的 Knoevenagel 缩合反应的可能机理 Scheme 3 Plausible mechanism of Knoevenagel condensation reaction catalyzed by EDAHS

SDS 形成的胶束能够在水溶液中溶解有机底物，以其作 为纳米反应器, 能够更好地溶解醛和活泼亚甲基化合 物, 促进其与 EDAHS 的充分接触. 该反应在水溶液中 进行，未使用金属或有机溶剂，操作简单，具有良好的 收率 $(80 \% \sim 96 \%)$. 可能的反应机理如 Scheme 4 所示: EDAHS 的酸性部分(铵盐部分)将芳香醛的碳基氧质子 化, 增加了羰基碳的电正性; 另一方面, EDAHS 的阴离 子部分具有碱性，可以去除活性亚甲基化合物的酸性质 子, 形成亲核试剂. 进而发生亲核加成得到含差基的中 间体，消除水得到最终的产物.

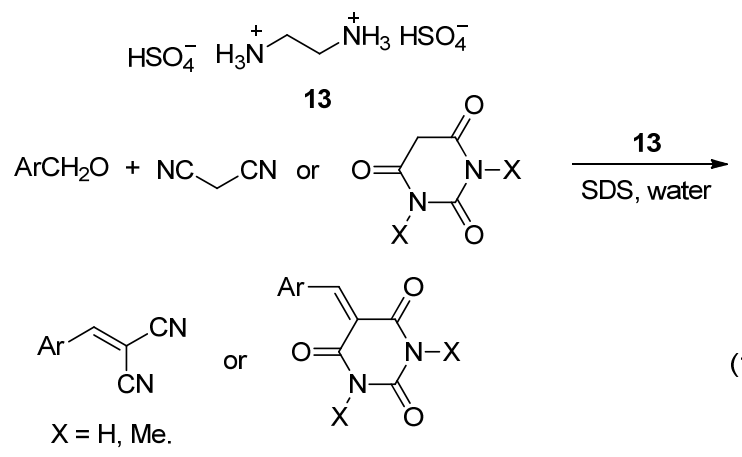

Shi 课题小组 ${ }^{[51]}$ 合成了聚合离子液体 1-[(4-甲基苯 基)甲基]-3-丙基咪唑基咪唑(BPIL, 14), 催化醛、酮与活 性亚甲基化合物的 Knoevenagel 缩合反应((Eq. 16). 因 为 BPIL 同时具有强碱性和高表面活性，故催化活性大 于普通的碱催化剂. 此外, 在苯甲醛、丙二腈反应体系 


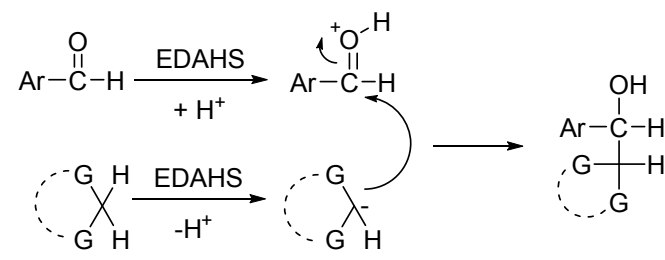

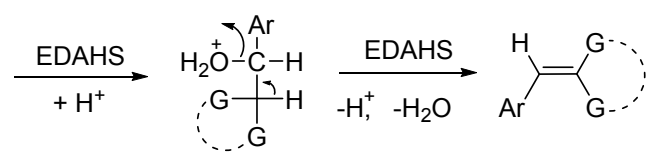

图式 4 EDAHS 参与的 Knoevenagel 缩合反应的机理

Scheme 4 Plausible catalytic pathway for the EDAHS-mediated Knoevenagel condensation

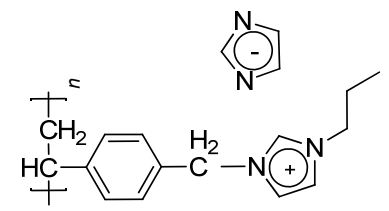

14

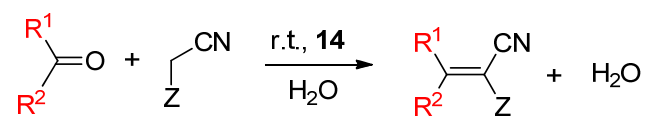

$\mathrm{Z}=\mathrm{CN}, \mathrm{COOEt}$

中, 离子液体 BPIL 简单回收后可重复使用 5 次并保持 高催化活性.

我们课题组 ${ }^{[52]}$ 以 DABCO 和 3-氯-1, 2-丙二醇为原 料合成了一系列新的碱性离子液体 $\mathbf{1 5}$, 用于催化芳香 醛与 $\alpha$-芳族(杂芳基或聚芳族)取代的亚甲基化合物的 Knoevenagel 缩合反应(Eq. 17). 该催化反应可在室温下 水中进行, 产物可直接从反应混合物中分离且催化剂可 循环使用 7 次而不丧失活性, 研究发现, [DABCO-PDO][OAc]催化活性最好, 收率高 $(73 \% \sim 96 \%)$. 以 苯甲醛和苯并噻唑-2-乙腈的反应为例, 文中提出了可 能的反应机理, 如 Scheme 5 所示: 一方面, 离子液体 $\mathrm{N}$ 原子上的孤对电子带走亚甲基上的氢原子形成活性碳 负离子; 另一方面, 离子液体的羟基可以和醛的羰基形 成氢键, 增强岸基碳的电正性, 促进碳负离子进攻羰基 碳; 因而起到了双重催化作用. 我们利用该催化剂制备 阿伐他汀钻重要中间体(图 2), 反应以水为溶剂, 室温反 应 $3 \mathrm{~h}$ ，高产率得到中间体(83\%).

Xing 研究小组 ${ }^{[53]}$ 也以 DABCO 为原料, 分别与 2氯乙氧基乙醇和 3-溴丙醇通过一步反应合成了两种羟 基功能化离子液体([DABCO-HEE]Cl, [DABCO-HPP]Br, 16)用于催化苯甲醛与氰基乙酸乙酯或 2,4-噻唑烷二酮 的 Knoevenagel 缩合反应(Eq. 18), 具有理想的收率 $(99 \%, 92 \%)$. 研究发现, [DABCO-HEE]Cl 催化效果优于 [DABCO-HPP]Br，在它的催化下，该反应在无溶剂，

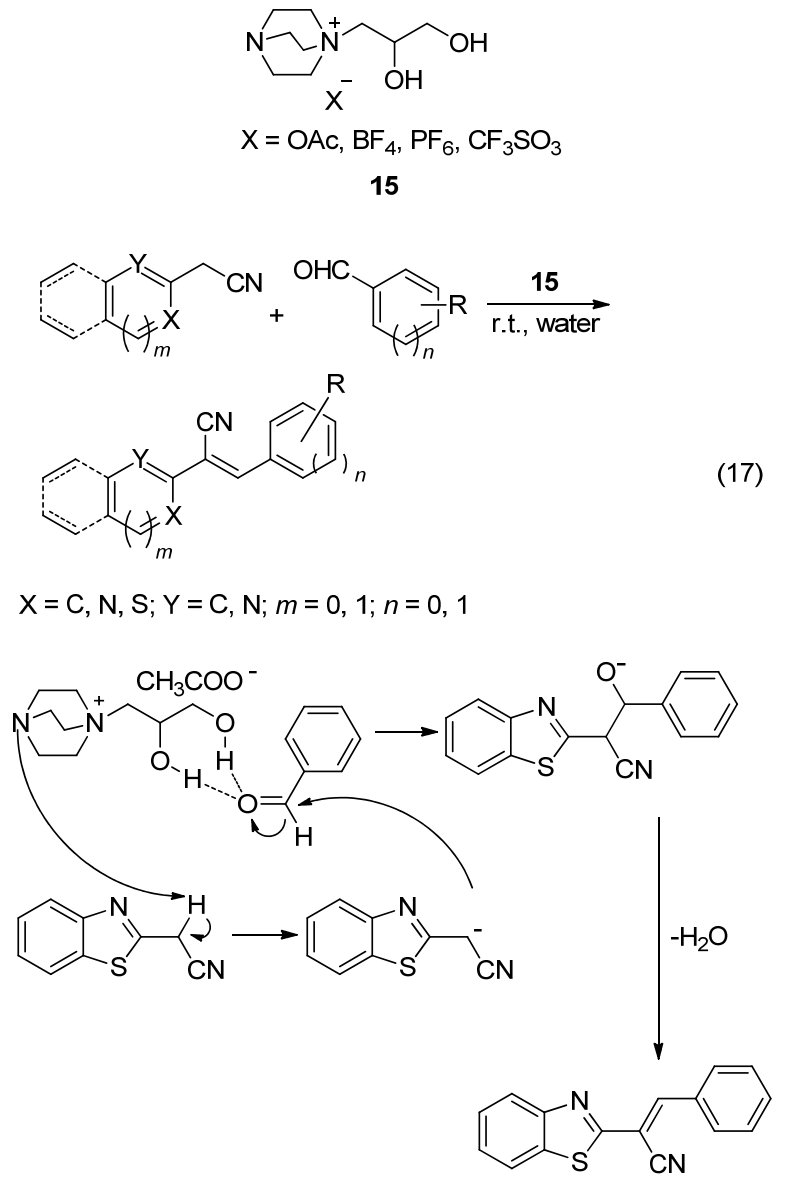

图式 5 [DABCO-PDO][OAc]催化的苯甲醛和苯并噻唑-2-乙 腈的反应机理

Scheme 5 Plausible mechanism for the reaction between benzaldehyde and benzothiazole-2-acetonitrile catalyzed by [DABCO-PDO][OAc]
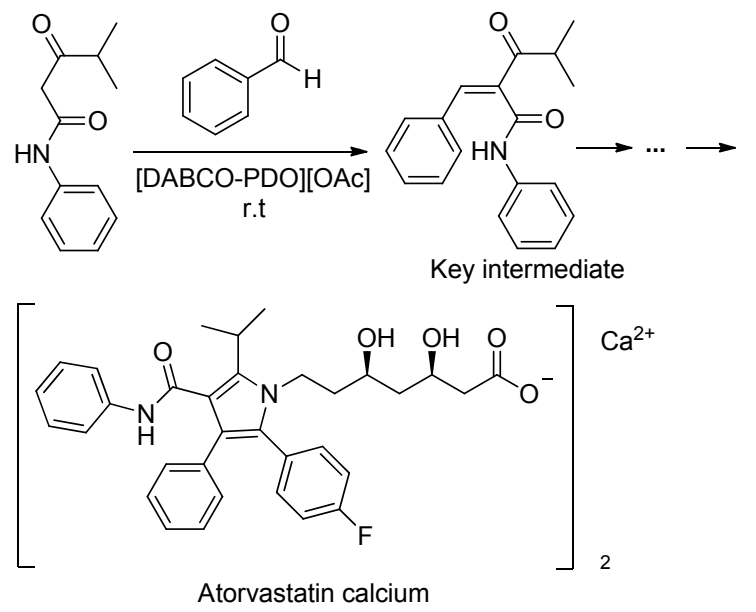

图 2 伐他汀钙的一种合成路线

Figure 2 A synthetic route of atorvastatin

$40{ }^{\circ} \mathrm{C}$ 的条件下反应 $20 \mathrm{~min}$, 收率分别达到 $99 \%$ 和 $94 \%$. 反应结束后，产物可从反应混合物中直接分离得到，并 且催化剂表现出较好的重复利用率, 可以至少重复使用 
4 次, 活性无明显下降.<smiles>OCCOCCN1CCN2CCN1C2</smiles><smiles>OCCCN1C2CCN1CC2</smiles>

[DABCO-HEE]Cl

$16 \mathrm{a}$

[DABCO-HPP]Br

$16 b$

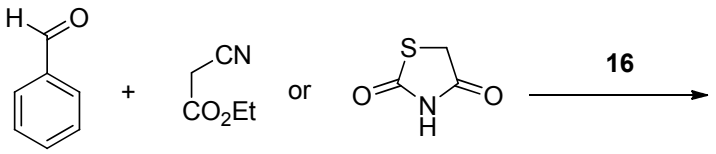<smiles>CCOC(=O)/C(C#N)=C\c1ccccc1</smiles>

\subsection{Aldol 缩合}

Davoodnia 等 ${ }^{[54]}$ 报道了酸性离子液体 1-(4-磺酸)丁 基吡啶硫酸氢盐([PYC $\left.\left.\mathrm{SO}_{3} \mathrm{H}\right]\left[\mathrm{HSO}_{4}\right], 17\right)$ 催化的芳香醛 与酮类的交叉 Aldol 缩合反应(Eq. 19). 他们利用二氧化 硅 $\left(\mathrm{A} 300 \mathrm{SiO}_{2}\right)$ 负载催化剂 17 , 反应结束后过滤即可回收 17, 催化剂可以至少重复使用 4 次, 活性稍微下降. 此 外, 该反应在无溶剂的条件下进行, 节约溶剂、环保且 不发生自缩合反应.<smiles></smiles>

17<smiles>CC(=O)C[C@H]1CCC[CH+](C(C)=O)C1</smiles>

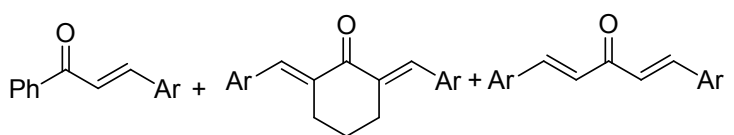

Gonzalez-Olmos 等 ${ }^{[55]}$ 合成了一系列 Brønsted 离子 液体 18 用于催化苯甲醛、3,7-二甲基-2,6-辛二烯醛和丙 酮的 Aldol 缩合反应(Eq. 20). 研究发现, 19 的催化效果 最好，苯甲醛与丙酮反应 $2 \mathrm{~h}$ 后，具有理想收率 $98.4 \%$, 3,7-二甲基-2,6-辛二烯醛与丙酮反应 $7 \mathrm{~h}$, 得到 $37.6 \%$ 的 收率. 反应结束后, 混合物中加入蒸馏水, 搅拌后静置 $15 \mathrm{~h}$, 水相中的离子液体经减压蒸馏回收, 可以重复使 用 3 次而没有明显的活性损失. 本研究中设计的离子液 体催化活性较其它体系并不是最好, 但它们易与反应介 质分离, 可多次循环使用, 符合绿色化学的理念, 可作 为方便、环保的绿色催化剂代替传统催化剂.
$\left(\mathrm{HOCH}_{2} \mathrm{CH}_{2}\right)_{x} \mathrm{NH}^{+}{ }_{y+1}\left(-\mathrm{OOC}\left(\mathrm{CH}_{2}\right)_{3} \mathrm{CH}_{3}\right)$

$$
x+y=3
$$

18

$\left(\mathrm{HOCH}_{2} \mathrm{CH}_{2}\right) \mathrm{NH}_{3}^{+}\left(-\mathrm{OOC}\left(\mathrm{CH}_{2}\right)_{3} \mathrm{CH}_{3}\right)$

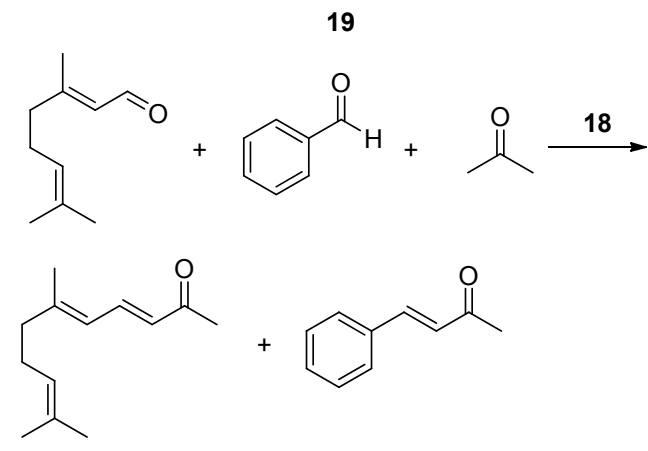

Gao 研究小组 ${ }^{[56]}$ 合成了季铵盐离子液体 $20 \sim 24$ 和 四丁基磷盐离子液体 $\mathbf{2 5} \sim \mathbf{2 6}$, 在微波辅助下催化芳香 醛和丙酮的 Aldol 缩合反应(Eq. 21). 研究发现, 20 催化 效果最优, 微波加热 $80{ }^{\circ} \mathrm{C}$, 反应 $30 \mathrm{~min}$, 收率达到 98\%, 转化率超过 99\%. 他们探讨了溶剂对该实验收率 的影响, 结果表明水是最佳的反应溶剂, 反应物溶于离 子液体一水介质中, 由氯仿萃取产物后, 收集剩余的水 和离子液体, 无需进一步处理即可催化下一个周期的反 应. 进一步研究发现, 该催化体系可至少重复使用 5 次<smiles>CC[N+](CC)(CC)CCNCCCS(=O)(=O)[O-]</smiles>

20

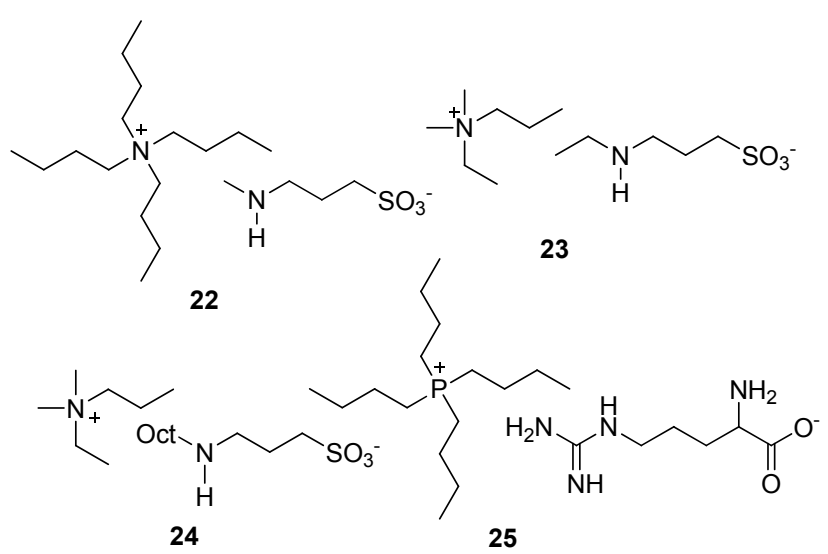

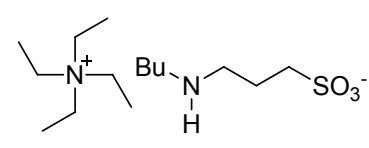

21 
并保持收率在 90\%左右, 甚至成功地拓展到了 Henry 反 应。<smiles></smiles><smiles>CC(=O)/C=C/c1ccccc1</smiles>

Wei 等 ${ }^{[57]}$ 合成了一系列新型二磺酸功能化的 Brønsted 酸离子液体 27 用于催化芳基醛和环酮的 Cross Aldol 缩合反应(Eq. 22), 并选择吗啉、甲基咪唑单磺酸 离子液体作为对照组, 以苯甲醛与环己酮为模板反应, 考察新型 ILs 的催化活性. 研究发现, 27 的催化活性高 于单磺酸离子液体, 且催化活性与 ILs 的酸性成正比, $\mathbf{A}$ 的催化效果最好, 收率可达 $91 \%$. 反应结束后, 将混合 液冷却结晶, 得到产品, 含离子液体的残留液经真空干 燥直接投入下一轮反应, 且重复使用 10 次仍具有较好 的催化活性.

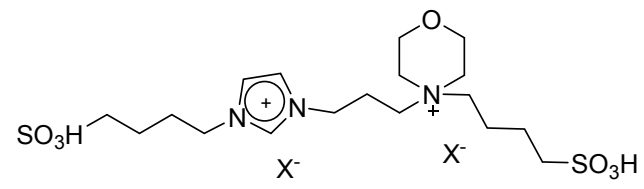

$X=O T f(\mathbf{A}), \mathrm{HSO}_{4}(\mathbf{B}), \mathrm{H}_{2} \mathrm{PO}_{4}(\mathbf{C})$

27

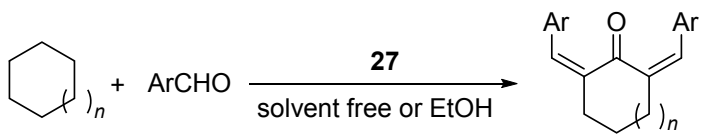

$n=0,1$

$\mathrm{Ar}=\mathrm{C}_{6} \mathrm{H}_{5}, 4-\mathrm{CH}_{3} \mathrm{OC}_{6} \mathrm{H}_{4}, 4-\mathrm{CH}_{3} \mathrm{C}_{6} \mathrm{H}_{4}, 4-\mathrm{HOC}_{6} \mathrm{H}_{4}, 4-\left(\mathrm{CH}_{3}\right)_{2} \mathrm{NC}_{6} \mathrm{H}_{4}$, $4-\mathrm{NO}_{2} \mathrm{C}_{6} \mathrm{H}_{4}, 4-\mathrm{FC}_{6} \mathrm{H}_{4}, 3-\mathrm{NO}_{2} \mathrm{C}_{6} \mathrm{H}_{4}, 2,4-\mathrm{Cl}_{2} \mathrm{C}_{6} \mathrm{H}_{3}$

我们课题组 ${ }^{[58]}$ 以廉价的 2 -氨基乙醇为原料合成了 一系列酸性离子液体 [SFHEA][X] (28)用于催化酫和硝 基烷烃的 Nitro Aldol 缩合反应(Eq. 23). 在 IL 的催化下, 该反应无需溶剂, 得到反式产物且有较高收率 $(91 \%)$. 反应结束后, 反应液经乙酸乙酯萃取, 然后真空蒸馏得 到粗产物并在乙醇中重结晶, 得到高纯度产物。萃取后 含离子液体的水相则在 $60{ }^{\circ} \mathrm{C}$ 的真空下蒸馏除水, 可以

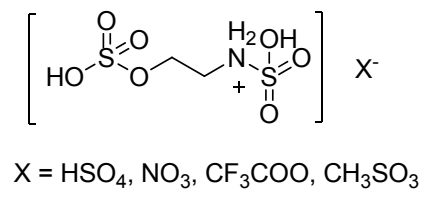

28

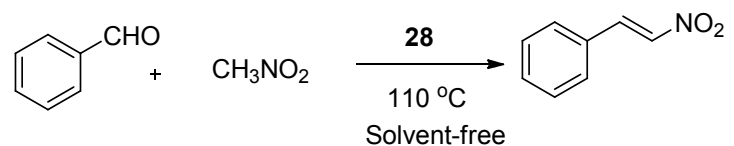

至少重复使用 6 次并保持收率基本不变. 我们课题组以 硝基甲烷和苯甲醛为例，提出了可能的反应机理，如 Scheme 6 所示: IL 与原料苯甲醛、硝基甲烷形成三重氢 键，增强醛的亲电性，同时活化硝基甲烷，得到 2-硝基1-羟基乙苯 $\mathbf{A}$. 随后, $\mathbf{A}$ 从催化剂得到一个质子形成中间 阳离子 $\mathbf{B}$ ，最后脱水得到产品.

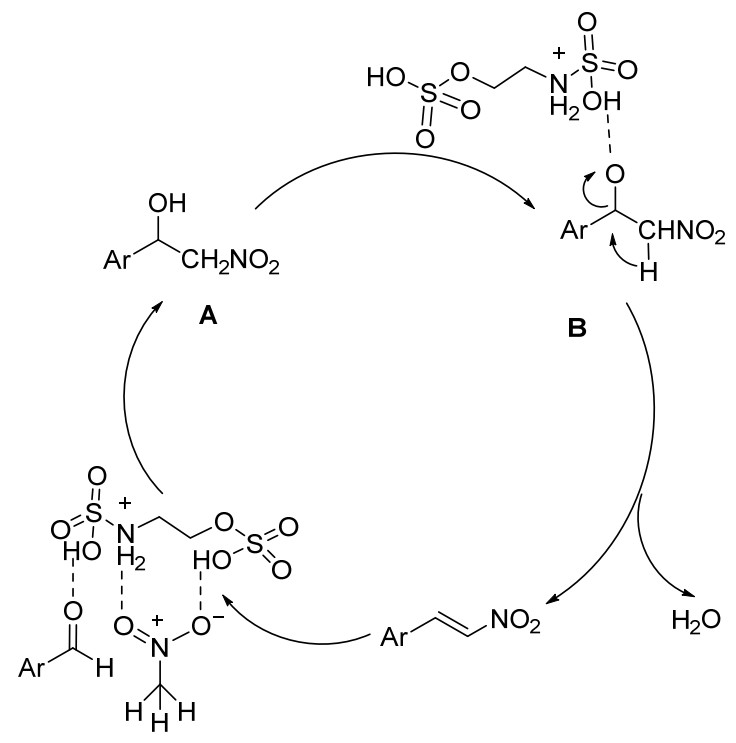

图式 6 离子液体 $[\mathrm{SFHEA}]\left[\mathrm{HSO}_{4}\right]$ 催化的 Nitro Aldol 反应机 理

Scheme 6 Proposed mechanism for the nitro aldol reaction catalyzed by [SFHEA] $\left[\mathrm{HSO}_{4}\right]$

\subsection{Diels-Alder 反应}

Chrobok 课题组 ${ }^{[59]}$ 设计合成了一类具有丰富氢键的 离子液体 $29 \sim 34$ 和 4 , 用于催化环戊二烯和马来酸二乙 酯及丙烯酸甲酯 Diels-Alder 反应(Eq. 24). 该反应的产 品是内型 $(e n d o)$ 和外型 $(e x o)$ 的混合物, 研究发现, 催化 剂中的烷基链越长, 选择性越差, 羟基数目越多, 催化 剂活性越好，故 $D$-葡萄糖系离子液体 $29 \sim 31$ 较其余离 子液体 32 34 和 4 活跃, 其中离子液体 29 为最优选择,
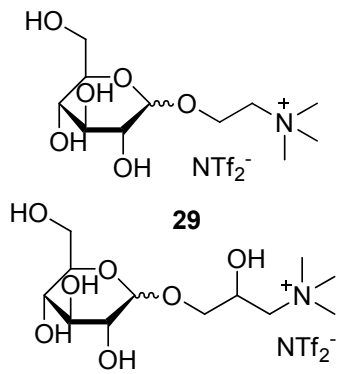

31<smiles>C[N+](C)(CCCO)CCCO</smiles>

33

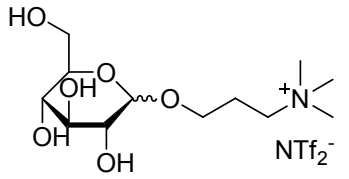

30<smiles>[15NH2]CC(O)CO</smiles>
$\mathrm{R}=\mathrm{CH}_{3}, \mathrm{CH}_{2} \mathrm{CH}_{3}, \mathrm{CH}_{2} \mathrm{CH}_{2} \mathrm{CH}_{3}$.

32<smiles>CCC[N+](C)(C)NCC</smiles> 


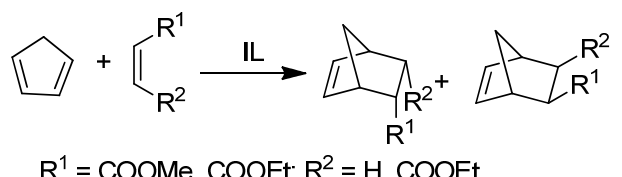

$\mathrm{R}^{1}=$ COOMe, COOEt; $\mathrm{R}^{2}=\mathrm{H}$, COOEt

除环戊二烯与甲基乙烯基酮外 $(85 \%)$, 温和条件下反应 $1 \mathrm{~h}$, 具有理想收率 $(90 \% \sim 99 \%)$. 离子液体在室温下以 液态形式存在, 热重分析结果显示其在高达 $430{ }^{\circ} \mathrm{C}$ 时 保持热稳定性.

$\mathrm{Liu}$ 等 ${ }^{[60]}$ 报道了氨基酸系离子液体丙氨酸甲酯硝酸 盐([AME] $\left.\left[\mathrm{NO}_{3}\right], 35\right)$ 催化的环龙二烯和甲基丙烯酸甲酯 之间的 Diels-Alder 反应(Eq. 25). 研究发现, [AME]$\left[\mathrm{NO}_{3}\right]$ 起到路易斯酸的作用, 其催化活性中心是 $[\mathrm{AME}]^{+}$阳离子, 它与甲基丙烯酸酯羰基上的氧形成 $\mathrm{N}-\mathrm{H} \cdots \mathrm{O}$ 的氢键, 使基丙烯酸酯的碳碳双键的极性增 强, 最终降低反应的能量势垒, 反应机理如 Scheme 7 . 进一步研究发现, 离子液体 35 催化该反应时, [AME ${ }^{+}$ 充当路易斯酸, 存在两对离子对有利于降低反应能量, 使空间位阻降低并形成更多的 $\mathrm{N}-\mathrm{H} \cdots \mathrm{O}$ 的氢键, 促进 反应的进行, 故该反应的两个反应物与催化剂的最佳摩 尔比为 $1: 1: 2$.<smiles>CC(=O)[C@H](C)[NH3+]</smiles>

35

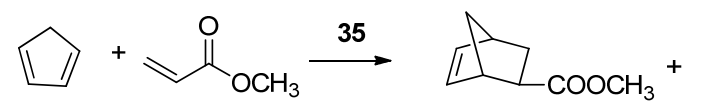<smiles>COC(=O)C1CC2C=CC1C2</smiles>

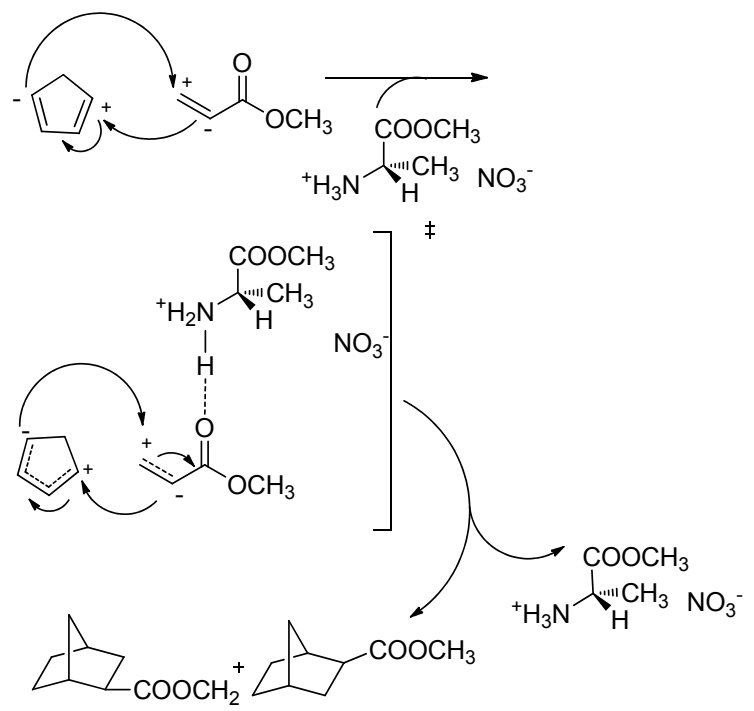

图式 7 离子液体 $[\mathrm{AME}]\left[\mathrm{NO}_{3}\right]$ 催化的 Diels-Alder 反应的机理 Scheme 7 Mechanism for the Diels-Alder reaction catalyzed by $[\mathrm{AME}]\left[\mathrm{NO}_{3}\right]$ ionic liquid

\section{4 偶联反应}

\subsection{Heck 反应}

Lee 等 ${ }^{[61]}$ 报道了以离子液体 $\mathbf{8}$ 为溶剂, 两性钯离子 络合物 36 催化的 Mizorokie-Heck 反应(Eq. 26). 该反应 在室温下进行 $12 \mathrm{~h}$, 可获得较高收率 $95 \%$, 且催化剂循 环使用 8 次, 仍然保持良好的催化活性.
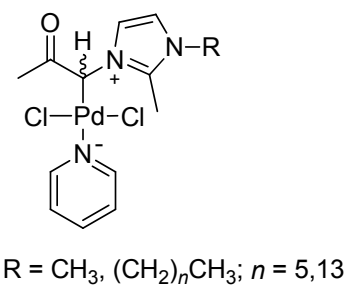

36

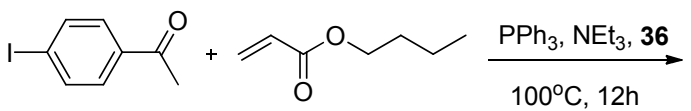<smiles>CCCCOC(=O)/C=C/c1ccc(C(C)=O)cc1</smiles>

Nowrouzi 课题组 ${ }^{[62]}$ 报道了以胺系离子性液体 $\left(\mathrm{R}_{3} \mathrm{~N}^{+}\right.$ $\mathrm{PPh}_{2}$, 37)为溶剂的 Heck 反应(Eq. 27), 以 $\mathrm{PdCl}_{2}$ 为催化 剂, 苯乙烯与芳基卤为底物, 在碱性条件下反应可获得 理想收率. 研究发现, 它具有高催化活性, $8 \mathrm{~min}$ 内产率 大于 $95 \%, \mathrm{NaOH}$ 为最适碱试剂, 比其他碱试剂高出 $\left(\mathrm{Et}_{3} \mathrm{~N} 、 \mathrm{Bu}_{3} \mathrm{~N}\right) 30 \% \sim 40 \%$. 反应后的催化剂和离子液体 可以通过简单的后处理分离回收, 可以至少重复使用 3 次，并且保持收率基本不变.

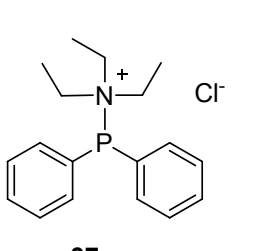

$37 a$<smiles>CCCC[N+](CCCC)(CCCC)P(c1ccccc1)c1ccccc1</smiles>

$37 b$<smiles>[X]c1ccc([R])cc1</smiles>

Luo 等 ${ }^{[63]}$ 研究了以离子液体 $([\mathrm{Bmim}] \mathrm{X}, 38)$ 和 $\mathrm{HBr}$ 的水溶液为溶剂, $\mathrm{PdBr}_{2}$ 催化的 Heck 反应(Eq. 28), 以炔 卤和 5-己烯-1-醇为底物, 在 $50{ }^{\circ} \mathrm{C}$ 下可以获得理想的收 率(94\%). 研究发现, [Bmim] $\mathrm{Br} / \mathrm{HBr}$ 具有较好的收率和 选择性，优化后较合适的反应条件为: 反应溶剂为 0.5 
$\mathrm{mL}[\mathrm{Bmim}] \mathrm{Br}$ 和 $0.25 \mathrm{~mL} \mathrm{HBr}$ 、反应温度为 $50{ }^{\circ} \mathrm{C}$. 该 路线条件温和, 易于操作处理, 为 $c i s-1,2$-二卤代烯烃类 化合物的合成提供了一条可行的路线.

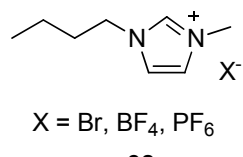

38
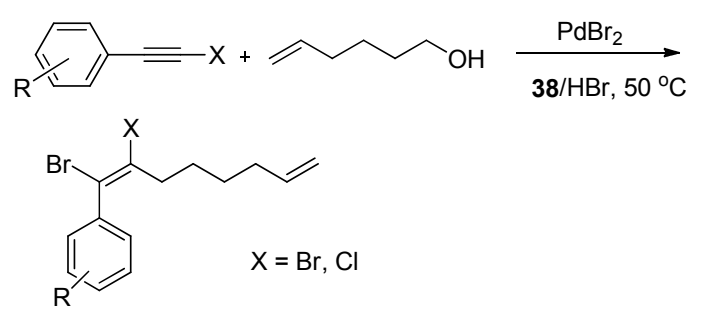

$\mathrm{R}=\mathrm{Ph}, 4-\mathrm{MeC}_{6} \mathrm{H}_{4}, 4-\mathrm{EtC}_{6} \mathrm{H}_{4}, 4-t-\mathrm{BuC}_{6} \mathrm{H}_{4}, 4-\mathrm{PhC}_{6} \mathrm{H}_{4}, 4-\mathrm{FC}_{6} \mathrm{H}_{4}$ 4- $\mathrm{ClC}_{6} \mathrm{H}_{4}, 4-\mathrm{BrC}_{6} \mathrm{H}_{4}, 2-\mathrm{ClC}_{6} \mathrm{H}_{4}, 2-\mathrm{BrC}_{6} \mathrm{H}_{4}, 4-\mathrm{MeOC}_{6} \mathrm{H}_{4}$, 4- $\mathrm{Me}_{2} \mathrm{C}_{6} \mathrm{H}_{3}$, 2-Thienyl

\subsection{Suzuki 反应}

Suzuki 反应是一类常用和有效的形成碳-碳键的方 法. 且该反应对官能团的耐受性非常好, 反应物可以带 着 $\mathrm{CHO} 、 \mathrm{COCH}_{3} 、 \mathrm{COOC}_{2} \mathrm{H}_{5} 、 \mathrm{OCH}_{3} 、 \mathrm{CN} 、 \mathrm{NO}_{2} 、 \mathrm{~F}$ 等 官能团进行反应而不受影响.

$\mathrm{Chu}$ 等 ${ }^{[64]}$ 研究了离子液体 7 为溶剂, 镍催化的芳基 卤与芳基嗍酸化合物的 Suzuki 交叉偶联反应(Eq. 29). 研究了不同溶剂、碱试剂和底物的影响, 结果显示, 该 催化剂具有高催化活性并可以回收再重复使用. 该实验 路线, 不仅获得了高收率的联苯衍生物(96.7\%), 且绿色 无污染, 离子液体在反应中作为溶剂, 减少了挥发溶剂 的使用，也方便回收利用.

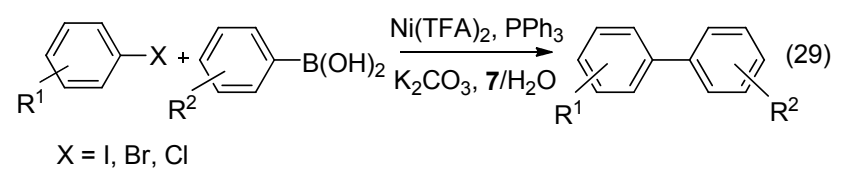

Sarma 研究小组 ${ }^{[6]}$ 合成了一系列离子液体 39, 形成 $\mathrm{IL} / \mathrm{LiCl}$ 体系用于催化芳基卤与芳基硼酸化合物的 Suzuki 反应(Eq. 30). 研究发现, [C C $_{4}$-DABCO][dca]的催 化效果最好, 产品收率可达 $90 \%$, 且反应可以在无碱、 无配体的条件下进行, 降低了反应的操作难度、节约了 成本. 此外, 反应终止, 用乙醚萃取产品后, 得到的催 化剂体系回收后可循环使用至少 4 次, 并保持催化活性 不变.

\section{5 不对称反应}

Zhang 等 ${ }^{[6]}$ 研究了离子液体负载的催化剂体系的应 用, 该课题组首先以咪唑类离子液体为原料, 通过简单 的二步反应合成了 1-[2-[2-(二苯膦基)二茂铁基]葝唑啉<smiles></smiles>

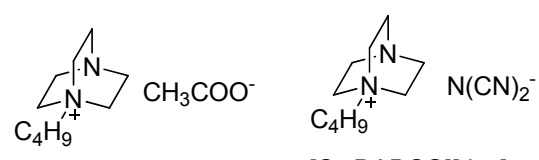

[C 4 -DABCO][Acetate]

$\left[\mathrm{C}_{4}\right.$-DABCO][dca]

39

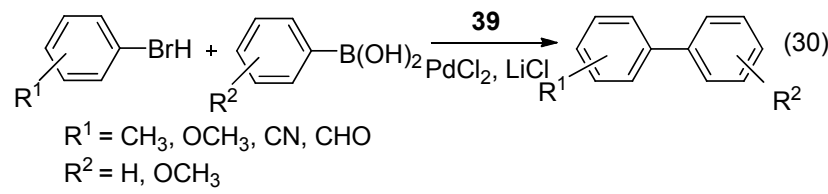

基]甲基季铵盐(40), 将 $\mathrm{RuCl}_{2}\left(\mathrm{PPh}_{3}\right)_{3}$ 负载于 $\mathbf{4 0}$, 得到负 载型催化剂 Ru-IL，参与芳族酮类化合物的不对称氢化 反应(Eq. 31). 研究发现, 以甲苯/ $\mathrm{H}_{2} \mathrm{O}$ 为溶剂, 在 $30{ }^{\circ} \mathrm{C}$ 的碱性条件下反应 $12 \mathrm{~h}$, 得到的不对称仲醇产物具有良 好的收率(99\%)和对映选择性(97.2\%). 反应结束后，回 收含有催化剂的水层, 无需后处理, 加入溶剂甲苯可以 直接循环利用. 该催化剂可以循环使用至少 4 次保持催 化活性和对映选择性基本不变.

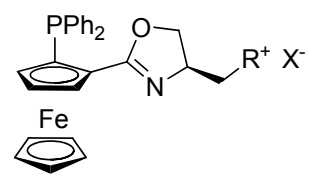

$\mathrm{R}=$ 1,2-dimethylimidazole $\mathrm{X}=\mathrm{OTf}, \mathrm{PF}_{6}, \mathrm{BF}_{4}$

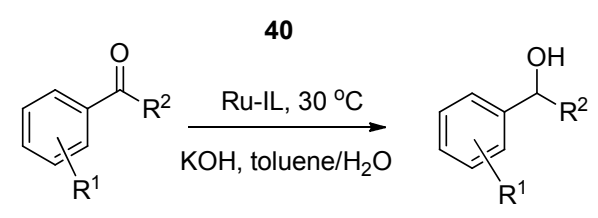

Singh 等 ${ }^{[67]}$ 利用离子液体合成配体 $\left((\mathrm{QN})_{2} \mathrm{PHAL}-\right.$ $\left.\left[\mathrm{C}_{5} \mathrm{mim}\right] \mathrm{Br}, 41\right)$, 随后负载于 $\mathrm{OsO}_{4}$-层状双氢氧化物 $\left(\mathrm{OsO}_{4}-\mathrm{LDH}\right)$ 得到多相催化剂, 用于催化不对称双差化 反应(Eq. 32). 以芳香族烯类化合物为底物, [bmim] $\mathrm{PF}_{6}$ $\mathrm{H}_{2} \mathrm{O}$-丙酮为溶剂, 反应条件温和, 室温下进行, 收率高 $(96 \%)$, 具有理想的立体选择性(94\%), 且催化剂可以回 收使用至少 5 次保持催化活性和对映选择性基本不变.

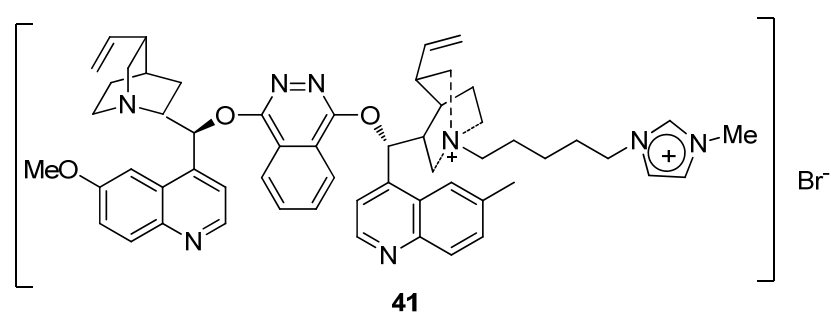




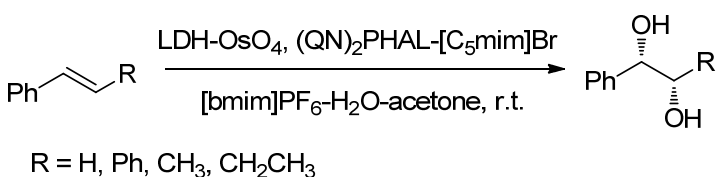

$\mathrm{Hou}$ 课题组 ${ }^{[68]}$ 以离子液体和钉盐为原料合成了一 系列催化剂 [PEG-2000- $\left.\mathrm{C}_{\mathrm{n}} \mathrm{mim}\right][\mathrm{Ru}-\mathrm{Ts}$ PPENDS] (42), 用于催化酮类化合物的不对称氢化反应(Eq. 33), 具有 理想的收率(99.7\%)和立体选择性(97\%). 研究发现, 42 的烷基咪唑阳离子拥有温控性能，以苯乙酮为模板反 应, $40{ }^{\circ} \mathrm{C}$ 时得到红棕色的均相反应液, 催化剂可以顺利 催化苯乙酮得到手性苯乙醇. 反应结束后, 降低温度, 催化剂从反应体系中完全沉淀, 简单倾析除去上层清 液, 即可回收 42, 循环使用 5 次而催化活性和对映选择 性保持基本不变.

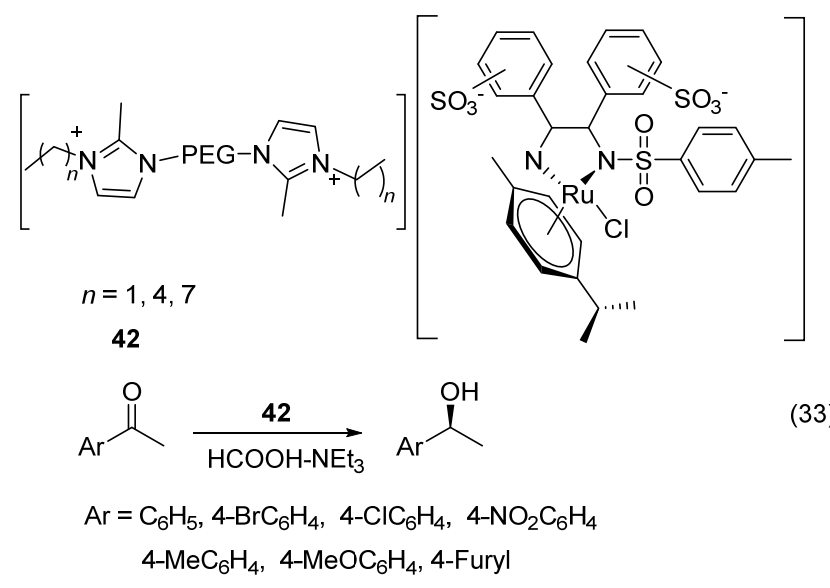

\section{6 多组分反应}

多组分反应(MCRs) 是指三个或三个以上的起始原 料进入反应，用 “一锅煮” 的方法最终生成一个终产 物, 在终产物结构中含有所有原料片段的合成方法. 多 组分化学反应具有操作简单、资源利用率高和高原子经 济性等特点, 是一类重要的有机化学反应, 在新药设计 与合成、组合化学和天然产物合成中具有广泛的应用.

\subsection{Biginelli 反应}

Aghakhanizadeh 等 ${ }^{[69]}$ 报道了 Brønsted 酸离子液体 $N$-甲基-2-吡咯烷酮磷酸二氢盐 $\left([\mathrm{NMP}] \mathrm{H}_{2} \mathrm{PO}_{4}, 43\right) 、 2$-吡 咯烷酮硫酸氢盐([Hnhp] $\left.\mathrm{HSO}_{4}, 44\right)$ 和 $N$-甲基-2-吡咯烷酮 硫酸氢盐([NMP] $\left.\mathrm{HSO}_{4}, 45\right)$ 催化的 Biginelli 反应(Eq. 34). 该系列离子液体可以在无溶剂的条件下, 有效催化芳香 醛、乙酸乙酯/乙酰乙酸甲酯和嫝/硫脲得到相应的 3,4二氢嘧啶-2(1H)-酮 (硫酮)的衍生物, 获得理想收率 $(80 \% \sim 85 \%)$. 反应结束后, 将反应混合液冷却到室温, 加入少量蒸馏水, 过滤得到粗产品, 用水洗涤两次. 水<smiles>CN1CCCC1=O</smiles>

$\left([\mathrm{NMP}] \mathrm{H}_{2} \mathrm{PO}_{4}\right.$

43<smiles>O=C1CCCS1(=O)=O</smiles>

$[\mathrm{HnhP}]\left[\mathrm{HSO}_{4}\right]$

44

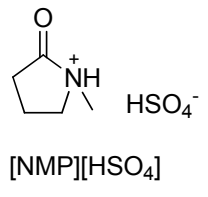

45
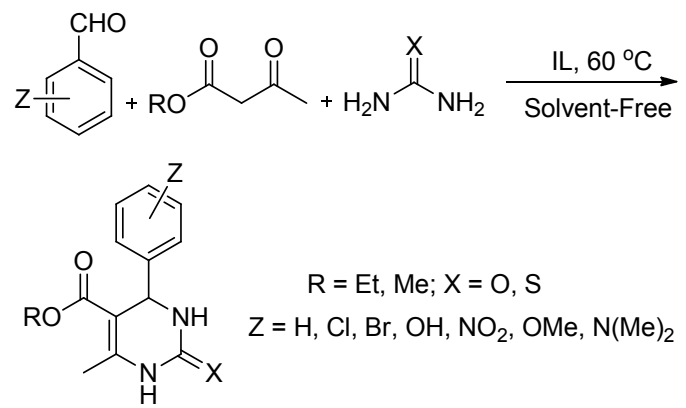

相中的离子液体蒸发浓缩后加入二氯甲烷，经减压蒸 馏、干燥可循环使用至少 5 次, 无明显的活性降低. 以 催化剂 $[\mathrm{Hnhp}] \mathrm{HSO}_{4}$ (44)为例, 反应机理如图 Scheme 8 所示: 首先, 醛与硫艮/艮在离子液体的催化下缩合得到 酰基亚胺化合物, 在酸性离子液体的条件下，质子化得 到中间体 $\mathbf{b}$, 然后与乙酰乙酸乙酯/甲酯的 $\mathrm{CH}$ 反应得到 中间体 $\mathbf{c}$. 随后 $\mathbf{c}$ 的羰基与嫝/硫艮的 $\mathrm{NH}_{2}$ 缩合，得到环 化的 Biginelli 产物.

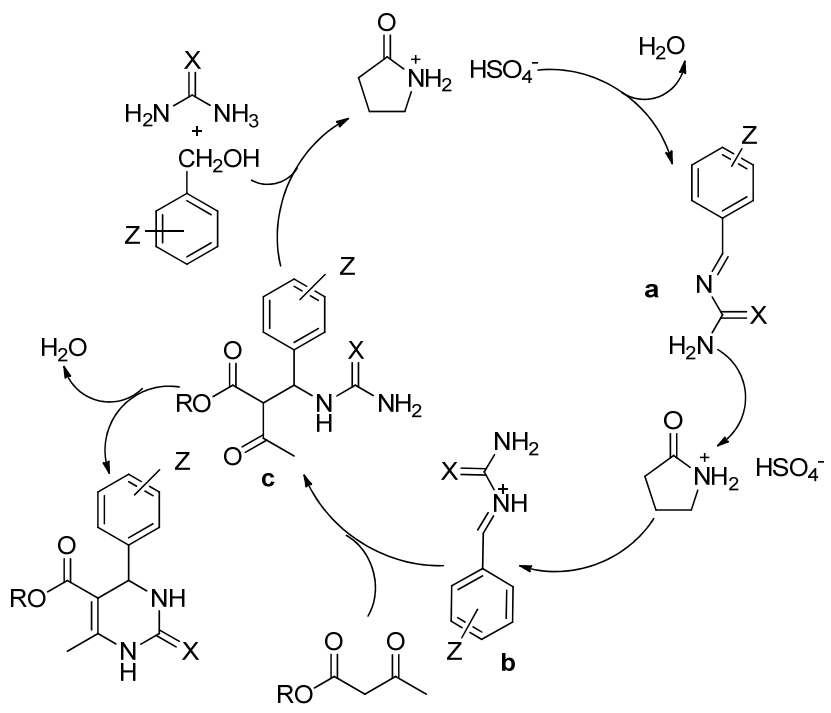

图式 8 以离子液体 $[\mathrm{Hnhp}] \mathrm{HSO}_{4}$ 为催化剂制备 3,4-二氢嘧 啶-2(1H)-酮(硫酮)衍生物的反应机理

Scheme 8 Proposed mechanism for $[\mathrm{Hnhp}] \mathrm{HSO}_{4}$ as selected ILs that catalyzed the preparation of 3,4-dihydropyrimidin-2(1H)-one (thione) derivatives

Liu 等 ${ }^{[70]}$ 通过两步原子经济法合成了一系列硫酸氢 盐离子液体(TSILS, 46)用于在水性介质中催化一锅煮 Biginelli 反应(Eq. 35). 将含有原料的苯甲醛、乙酰乙酸 乙酯和尿素的混合溶液直接在 $90{ }^{\circ} \mathrm{C}$ 下反应 $60 \mathrm{~min}$ 不能 
得到任何产物，表明该催化剂是必要的，同时，催化剂 的最适用量为 $2 \mathrm{~mol} \%$, 再增加催化剂的用量对收率无 明显影响. 产品简单分离后, 含有离子液体的滤液无需 进行任何处理, 即可通入下一次反应, 可循环使用 6 次, 活性基本保持不变.

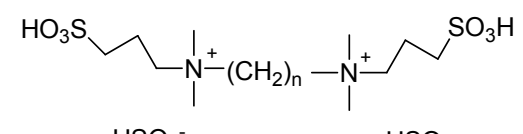
$\mathrm{HSO}_{4}^{-} \quad n=2,3,6 \quad \mathrm{HSO}_{4}^{-}$<smiles>[R]C(=O)CC([R])=O</smiles>

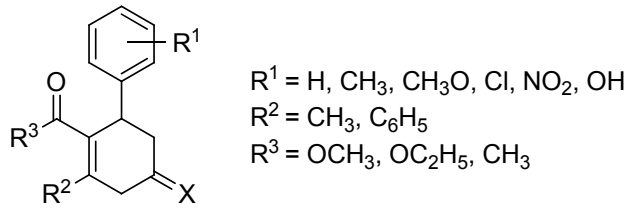

\subsection{Mannich 反应}

Zhao 研究小组 ${ }^{[7]}$ 合成了一系列双取代 Brønsted 酸 离子液体 GBAILs (47), 用于催化水溶液中芳香醛、酮 和胺类化合物的 Mannich 反应(Eq. 36). 他们利用苯甲 醛、环已酮和苯胺为模板反应, 考察了离子液体的作用. 在反应中离子液体发挥着催化剂的作用, 侧链烷烃含有 14 个 $\mathrm{C}$ 的催化剂 GBAIL-C14 活性高, 催化效果最好, 在室温下即可获得理想的收率 $(85 \% \sim 94 \%)$, 并且该催 化剂连续使用 7 次后催化活性无明显变化.<smiles>Cc1ccc(S(=O)(=O)O[Na])cc1</smiles>

$n=4,8,10,12,14,16,18$

47<smiles></smiles>

$\mathrm{R}^{1}=\mathrm{H}, p-\mathrm{Cl}, p$-OMe, o-OMe

$\mathrm{R}^{2}=\mathrm{H}, p-\mathrm{OMe}, o-\mathrm{NO}_{2}, o-\mathrm{Cl}$

Kandasamy 等 ${ }^{[72]}$ 合成了一系列 $N$-烷基-1,2,4-三唑甲 磺酸盐类离子液体 48 , 用于催化乙醇介质中的三组分 Mannich 反应(Eq. 37). 反应结束后, 将少量的水加入到 混合物中, 过滤得到粗产品, 滤液中加入少量乙醇, 真 空旋转蒸发后, 即可投入下一次反应, 可以至少循环使 用 4 次, 活性无明显变化.

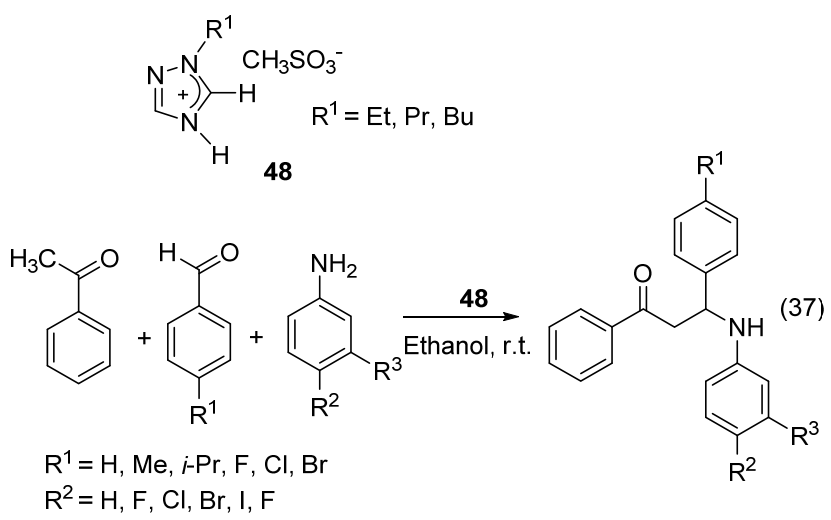

\subsection{Hantzsch 反应}

Deshmukh 课题组 ${ }^{[73]}$ 报道了新型酸性离子液体 3-(羧甲基)-1-甲基 - $1 H$ - 咪唑 -3-鎓三氟乙酸 $\left([\mathrm{Cmim}] \mathrm{CF}_{3} \mathrm{COO}, 49\right)$ 催化的酮、醛和胺的多组分 Hantzsch 反应(Eq. 38). 研究发现, 该催化剂适用于水介 质, 最适溶剂为乙醇和水的体积比为 $1: 1$ 的混合溶液, 相同条件下收率比传统溶剂高出 $10 \%$ ～ $50 \%$, 且对环境 无污染符合绿色化学的要求. 反应结束后, 通过简单的 处理得到粗产品, 无需过柱, 简化了操作, 得到含有催 化剂 46 的滤液经乙醚萃取, 减压蒸馏, 干燥后可循环使 用, 并且重复利用 4 次后, 活性无明显下降. 反应机理 如 Scheme 9 所示: 首先，一分子原料酮的一个羰基与离 子液体 46 形成氢键, 得到烯醇结构的中间体 a. 随后 $\mathbf{a}$ 与苯甲醛经过 Knoevenagel 缩合并脱去一分子水得到 $\mathbf{b}$, 同时另一分子原料酮与 $\mathrm{NH}_{4} \mathrm{OAc}$ 反应脱去一分子水得 到烯胺 $\mathbf{c}$. 最后, $\mathbf{b}$ 和 $\mathbf{c}$ 分子间成环, 脱去水得到产物.<smiles></smiles><smiles>CC1(C)CC(=O)CC(C)(C)C(=O)C1</smiles>

Davoodnia 等 ${ }^{[74]}$ 报道了 Brønsted 离子液体 1-(4-磺酸 基)丁基-3-甲基咪唑硫酸氢盐([( $\left.\left(\mathrm{CH}_{2}\right)_{4} \mathrm{SO}_{3} \mathrm{Hmim}\right] \mathrm{HSO}_{4}$, 50)催化的 Hantzsch 反应(Eq. 39). 他们利用苯甲醛、环 己二酮和乙酰乙酸乙酯为模板反应, 研究了该反应. 结 果显示，该反应中无离子液体时收率仅有 $30 \%$ 并伴随着 


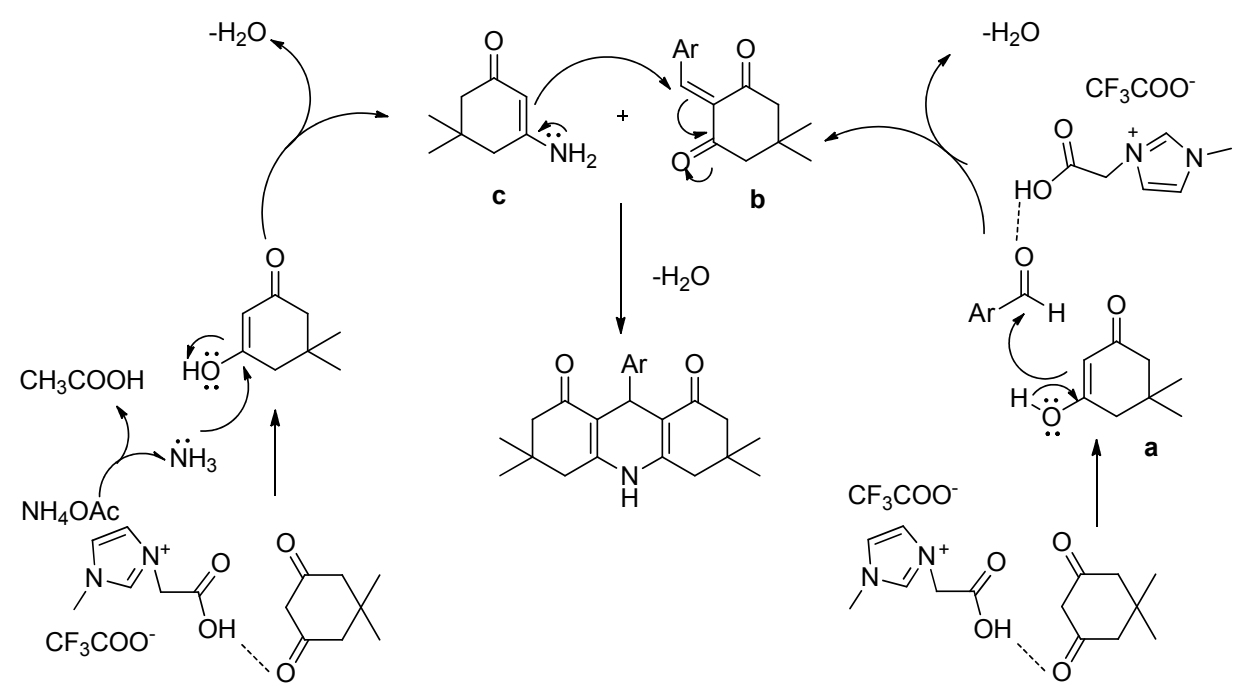

图式 $9[\mathrm{Cmim}] \mathrm{CF}_{3} \mathrm{COO}$ 催化的 Hantzsch 反应机理

Scheme 9 Pausible mechanism for the Hantzsch reaction catalyzed by $[\mathrm{Cmim}] \mathrm{CF}_{3} \mathrm{COO}$

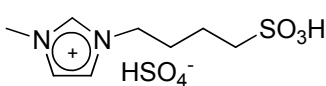

50

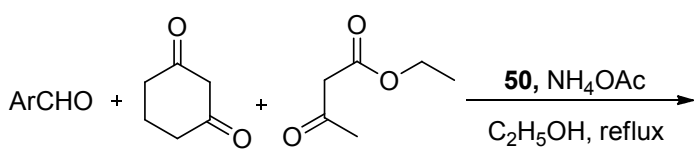<smiles>CCOC(=O)C1=C(C)NC2=C(C(=O)CCC2)C1Br</smiles>

副反应，极性溶剂有助于反应的进行，在反应中 47 发挥 着催化剂的作用，在乙醇和回流条件下反应可得 $90 \%$ 的 收率. 在反应结束后, 过滤得到的催化剂用乙醚洗涤, 在 $50{ }^{\circ} \mathrm{C}$ 下真空干燥下 $1 \mathrm{~h}$, 即可投入下一个反应循环利 用.

我们课题组 ${ }^{[75]}$ 利用离子液体催化的三组分反应合 成了 $\alpha$-氨基膦酸酯, 在酸性离子液体([SFHEA $][X], 28)$ 催化下, 无需溶剂使用芳醛、亚磷酸二乙酯和芳族胺一 锅法合成 $\alpha$-氨基膦酸酯(Eq. 40). 研究发现, 该系列催化 剂反应效果好, 时间短且收率高 $(91 \%)$, 后处理简单. 反 应结束后, 用乙酸乙酯萃取产物, 真空减压旋蒸后在乙 醇中重结晶, 得到高纯度产品, 含有催化剂的水相经过 简单真空旋蒸后，可直接投入下一个反应，循环使用 5 次后, 催化效果无明显下降. 反应机理如 Scheme 10 所 示: 芳醛的氧与 IL 形成氢键增加醛的亲电性, 然后 $\mathrm{N}$ 上 的孤对电子进攻羰基碳得到 $\mathbf{A}$. 随后, $\mathbf{A}$ 与 IL 通过氢键 生成中间体 B, B 脱水得到 C. C 和亚磷酸二乙酯分别与 IL 形成氢键, 增强了 $\mathbf{C}$ 的活泼性和亚磷酸二乙酯的亲核 性，亚磷酸二乙酯进攻 $\mathbf{C}$ 得到产物.<smiles>[X]CCOS(=O)(=O)OCCNS(=O)(=O)O</smiles><smiles>[R]CO[R]O[Y16]#N</smiles>

$\mathrm{R}^{1}=\mathrm{C}_{6} \mathrm{H}_{5}, 2-\mathrm{CH}_{3} \mathrm{OC}_{6} \mathrm{H}_{4}, 3-\mathrm{CH}_{3} \mathrm{OC}_{6} \mathrm{H}_{4}, 4-\mathrm{CH}_{3} \mathrm{OC}_{6} \mathrm{H}_{4}, 4-\mathrm{HOC}_{6} \mathrm{H}_{4}$, 3- $\mathrm{NO}_{2} \mathrm{C}_{6} \mathrm{H}_{4}, 4-\mathrm{NO}_{2} \mathrm{C}_{6} \mathrm{H}_{4}$

$\mathrm{R}^{2}=\mathrm{C}_{6} \mathrm{H}_{5}, 3-\mathrm{CH}_{3} \mathrm{C}_{6} \mathrm{H}_{4}, 2-\mathrm{CH}_{3} \mathrm{C}_{6} \mathrm{H}_{4}, 4-\mathrm{ClC}_{6} \mathrm{H}_{4}, 4-\mathrm{EtOC}_{6} \mathrm{H}_{4}$, $4-\mathrm{NO}_{2} \mathrm{C}_{6} \mathrm{H}_{4}, 4-\mathrm{OCH}_{3} \mathrm{C}_{6} \mathrm{H}_{4}$

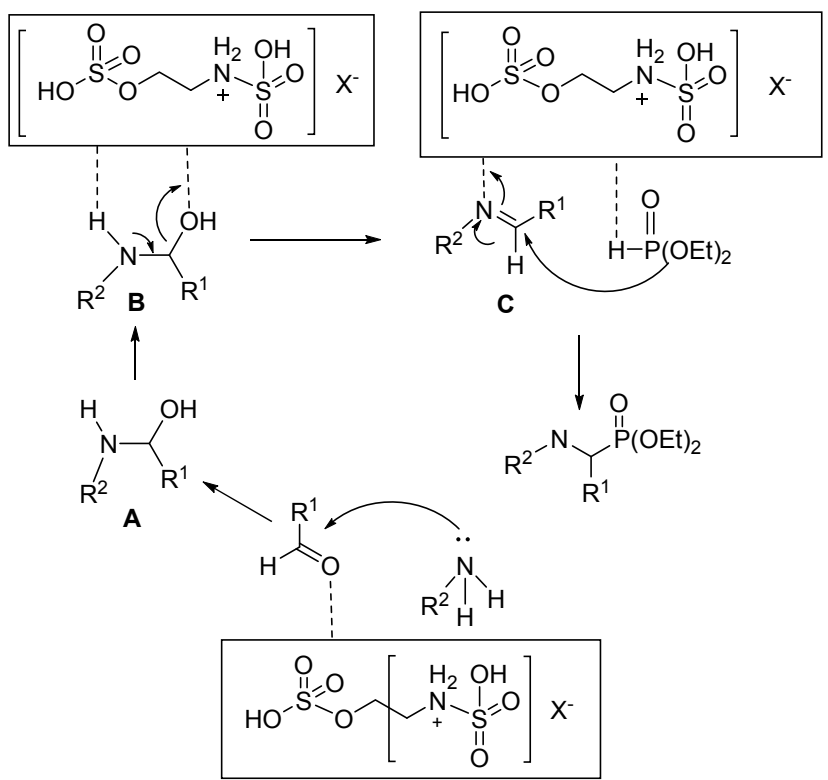

图式 10 [SFHEA] $\left[\mathrm{HSO}_{4}\right]$ 催化合成 $\alpha$-氨基膦酸酯的机理图 Scheme 10 Proposed mechanism for the synthesis of $\alpha$-aminophosphonates promoted by [SFHEA] $\left[\mathrm{HSO}_{4}\right]$ 


\section{7 结语}

综上所述, 离子液体作为催化剂或溶剂在有机合成 领域已有广泛的运用，包括加成、Knoevenagel 缩合、氧 化还原反应、偶联反应和多组分反应等. 离子液体作为 催化剂大多具有收率高、可回收循环使用多次并保持较 高活性的优点. 相较大部分传统的催化剂, 离子液体稳 定性好, 易与产品分离, 可以多次循环使用, 有利于提 高经济效益. 此外，离子液体还可以充当 Lewis 酸碱、 反应溶剂, 避免了强酸、强碱以及一些有毒有害有机溶 剂在反应中的使用, 减少环境污染. 然而, 在现代工艺 中, 部分离子液体的制备技术并没有十分成熟，且成本 较高. 所以我们未来应当在离子液体的设计、合成和应 用方面深入研究、寻求突破，使离子液体在工业化有机 化工合成中拥有更美好的前景.

\section{References}

[1] Yi, F. P.; Zhang, X.; Sun, H. Y.; Chen, S. H. Acta Chim. Sinica 2012, 70, 741 (in Chinese). (易封萍，张旋，孙海洋，陈世洪，化学学报, 2012, 70, 741.)

[2] Rogers, R. D. Nature 2007, 447, 917.

[3] Poulimenoua, N. I.; Giannopouloua, I.; Paniasa, D. Mater. Manuf. Process 2015, 30, 1403.

[4] Qureshi, Z. S.; Deshmukh, K. M.; Bhanage, B. M. Clean. Technol. Environ. Policy 2014, 16, 1487.

[5] Yang, J. B.; Zhou, L. H.; Guo, X. T.; Li, L. Chem. Eng. J. 2015, $280,147$.

[6] Zhou, Y.; Huang, W.; Chen, X. S.; Song, Z. B.; Tao, D. J. Catal. Lett. 2015, 145, 1830.

[7] Ali, E.; Alnashef, I.; Ajbar, A.; Mulyono, S.; Hizaddin, H. F.; Hadj-Kali, M. K. Korean J. Chem. Eng. 2013, 30, 2068.

[8] Xu, S. Y.; Zhang, F. X.; Li, J. Y.; Bai, Y.; Xiao, W. J.; Peng, J. J. Prog. Chem. 2015, 27, 1400 (in Chinese). (徐艺松, 张凤香, 厉嘉云, 白赢, 肖文军, 彭家建, 化学进展, 2015, 27, 1400.)

[9] Benzagouta, M. S.; AlNashef, I. M.; Karnanda, W.; Al-Khidir, K. Korean J. Chem. Eng. 2013, 30, 2108.

[10] Mai, N. L.; Kim, S. H.; Ha, S. H.; Shin, H. S.; Koo, Y. M. Korean J. Chem. Eng. 2013, 30, 1804.

[11] Tseng, M. C.; Liang, Y. M.; Chu, Y. H. Tetrahedron Lett. 2005, 46, 6131.

[12] Zang, H. H.; Zhang, Y.; Cheng, B. W.; Zhang, W. W.; Xu, X. L.; Ren, Y. L. Chin. J. Chem. 2012, 30, 362.

[13] Ma, Y. Q.; Wang, R. Chem. J. Chin. Univ. 2013, 35, 760 (in Chinese).

(马云倩, 王睿, 高等学校化学学报, 2013, 35, 760.)

[14] Popescu, A. M.; Constantin, V. Chem. Res. Chin. Univ. 2014, 30, 119

[15] (a) Vander, H. T.; Wellens, S.; Verachtert, K.; Binnemans, K. Green Chem. 2013, 15, 919.

(b) Clark, K. D.; Nacham, O.; Yu, H. L.; Li, T. H.; Yamsek, M. M.; Ronning, D. R.; Anderson, J. L. Anal. Chem. 2015, 87, 1552.

[16] Abbott, A. P.; Frisch, G.; Gurman, S. J.; Hillman, A. R.; Hartley, J.; Holyoak, F.; Ryder, K. S. Chem. Commun. 2011, 47, 10031.

[17] Tschan, M. J. L.; Dieboh, O.; Leeuwen, P. W. N. M. Top. Catal. 2014, 57, 1054 .
[18] Oh, Y.; Hu, X. L. Chem. Commun. 2015, 51, 13698.

[19] Gallardo, I.; Vila, N. J. Org. Chem. 2010, 75, 680.

[20] Hsieh, Y. T.; Sun, I. W. Chem. Commun. 2014, 50, 246.

[21] Armand, M.; Endres, F.; Macfarlane, D. R.; Ohno, H.; Scrosati, B. Nat. Mater. 2009, 8, 621 .

[22] Wang, C. F.; Chen, Y. J.; Zhuo, K. L. Chem. Commun. 2013, 49, 3336.

[23] Hou, H. L.; Li, Z. F.; Ying, A. G.; Xu, S. L. Chin. J. Org. Chem. 2014, 34, 1277 (in Chinese).

(侯海亮, 李志峰, 应安国, 许松林, 有机化学, 2014, 34, 1277. )

[24] Hou, H. L.; Li, Z. F.; Ying, A. G.; Xu, S. L. Chin. J. Org. Chem. 2014, 34, 1074 (in Chinese).

(李志峰, 侯海亮, 应安国, 许松林, 有机化学, 2014, 34, 1074.)

[25] Pan, L. Y.; Li, Z. F.; Ni, Y. X.; Yao, Z. G.; Yu, Z. P.; Wu, W. K.; Ying, A. G. Chem. J. Chin. Univ. 2015, 36, 81 (in Chinese). (泮丽亚, 李志峰, 倪宇翔, 姚振刚, 余志平, 吴文康, 应安国, 高等学校化学学报, 2015, 36, 81.)

[26] (a) Basavaiah, D.; Dharma, R. P.; Suguna, H. R. Tetrahedron 1996, 52,8001 .

(b) Basaviah, D.; Rao, A. J.; Satyanarayana, T. Chem. Rev. 2003, $103,811$.

[27] Zhao, S. H.; Zhang, Q. J.; Duan, X. E. Synth. Commun. 2011, 41, 3289.

[28] Zhao, S. H.; Wang, D.; Wang, M.; Kang, J.; Zhang, L. W.; Chin. J. Org. Chem. 2015, 35, 865 (in Chinese). (赵三虎, 王豆, 王敏, 康锦, 张立伟. 有机化学, 2015, 35, 865.)

[29] Song, Y.; Ke, H. H.; Wang, N.; Wang, L. M.; Zou, G. Tetrahedron 2009, 65, 9086.

[30] Lenardão, E. J.; Feijó, J. O.; Thurow, Samuel.; Perin, G.; Jacob, R. G.; Silveira, C. C. Tetrahedron Lett. 2009, 50, 5215.

[31] Keithellakpam, S.; Laitonjam, W. S. Chin. Chem. Lett. 2014, 25, 767.

[32] Chelghoum, M.; Bahnous, M.; Bouraiou, A.; Bouacida, S.; Belfaitah, A. Tetrahedron Lett. 2012, 53, 4059.

[33] Ying, A. G.; Li, Z. F.; Yang, J. G.; Liu, S.; Xu, S. L.; Yan, H.; Wu, C. L. J. Org. Chem. 2014, 79, 6510.

[34] Hou, H. L.; Qiu, F. L.; Ying, A. G.; Xu, S. L. Chin. Chem. Lett. 2015, 26, 377

[35] Du, Z. Y.; Ng, H. F.; Zhang, K.; Zeng, H. Q.; Wang, J. Org. Biomol. Chem. 2011, 9, 6930 .

[36] Van, Doorslaer. C.; Schellekens, Y.; Mertens, P.; Binnemansb, K.; De, Vos. D. Phys. Chem. Chem. Phys. 2010, 10, 1741

[37] Sun, W. Y.; Ueyama, N.; Nakamura, A. Tetrahedron 1993, 49, 1357.

[38] Nishiguchi, T.; Asano, F. J. Org. Chem. 1989, 54, 1531.

[39] Sahiner, N.; Sagbas, S.; Nahit, A. RSC Adv. 2015, 5, 18183.

[40] Das, J.; Velusamy, P. J. Taiwan Inst. Chem. Eng. 2014, 45, 2280.

[41] Sahiner, N.; Kaynak, A.; Butun, S. J. Non-Cryst. Solids 2012, 358, 758.

[42] Chauhan, M. S.; Singh, S. J. Mol. Catal. A: Chem. 2015, 398, 184.

[43] Chinnappan, A.; Kim, H. RSC. Adv. 2013, 3, 3399.

[44] Freeman, F. Chem. Rev. 1980, 80, 329

[45] Tietze, L. F. Chem. Rev. 1996, 96, 115

[46] Rumer, J. W.; Levick, M.; Dai, S. Y.; Rossbauer, S.; Huang, Z. G.; Biniek. L.; Anthopoulos, T. D.; Durrant, J. R.; Procter, D. J.; McCulloch, I. Chem. Commun. 2013, 49, 4465.

[47] Liang, F.; Pu, Y. J.; Kurata, T.; Kido, J.; Nishide, H. Polymer 2005 46, 3767.

[48] Zahouily, M.; Salah, M.; Bahlaouane, B.; Rayadh, A.; Houmam, A.; Hamed, E. A.; Sebti, S. Tetrahedron 2004, 60, 1631.

[49] Ouyang, F.; Zhou, Y.; Li, Z M.; Hu, N.; Tao, D. J. Korean J. Chem. Eng. 2014, 31, 1377.

[50] Bahrami, K.; Khodaei, M. M.; Babajani N.; Naali, F. J. Iran. Chem. 
Soc. 2014, 11, 1675 .

[51] Ding, L. B.; Li, H. S.; Zhang, Y. P.; Zhang, K.; Yuan, H.; Wu, Q.; Zhao, Y.; Jiao, Q. Z.; Shi, D. X. RSC Adv. 2015, 5, 21415.

[52] Ying, A. G.; Ni, Y. X.; Xu, S. L.; Liu, S.; Yang, J. G.; Li, R. R. Ind. Eng. Chem. Res. 2014, 53, 5678.

[53] Liu, Y. T.; Li, R.; Xing, Y. J. Chin. J. Org. Chem. 2015, 35, 1520 (in Chinese).

(刘玉婷, 李戎, 邢彦军, 有机化学, 2015, 35, 1520.)

[54] Davoodnia, A.; Yassaghi, G. Chin. J. Catal. 2012, 33, 1950.

[55] Iglesias, M.; Gonzalez-Olmos, R.; Cota, I.; Medina, F. Chem. Eng. J. 2010, 162, 802 .

[56] Wang, C.; Liu, J.; Leng, W. G.; Gao, Y. N. Int. J. Mol. Sci. 2014, 15, 1284.

[57] Cui, W. H.; Zhang, Y. M.; Jia, R. Y.; Wang, Y.; Wei, T. B. Chin. J. Org. Chem. 2015, 35, 890 (in Chinese).

(崔文辉, 张有明, 贾如琰, 王昱, 魏太保, 有机化学, 2015, 35, 890.)

[58] Ying, A. G.; Xu, S. L.; Liu, Shuo.; Ni, Y. X.; Yang, J. G.; Wu, C. L. Ind. Eng. Chem. Res. 2014, 53, 547.

[59] Erfurt, K.; Wandzik, I.; Walczak, K.; Matuszek, K.; Chrobok, A. Green Chem. 2014, 16, 3508.

[60] Zhu, X. Y.; Liu, J. Q.; Zhang, D. G.; Liu, C. B. Comput. Theor. Chem. 2012, 996, 21.

[61] Wang, Y. P.; Lee, H. M. J. Org. Chem. 2015, 791, 90.

[62] Nowrouzia, N.; Tarokha, D.; Motevalli, S. J. Mol. Catal. A: Chem. 2014, 385, 13.
[63] Yang, S. R.; Ling, G. W.; Ma, J. Y.; Zhang, L.; Li, J. X.; Luo, W. Chin. J. Org. Chem. 2014, 34, 1148 (in Chinese).

(杨少容, 凌厂围, 马建业, 张蕾, 李建晓, 罗维, 有机化学, 2014, 34, 1148.)

[64] Wang, M.; Yuan, X. B.; Li, H. Y.; Ren, L. M.; Sun, Z. Z.; Hou, Y. J.; Chu, W. Y. Catal. Commun. 2015, 58, 154.

[65] Boruah, P. R.; Koiri, M. J.; Bora, U.; Sarma, D. Tetrahedron Lett. 2014, 55, 2423.

[66] Xu, D.; Zhou, Z. M.; Da, L.; Tang, L. W.; Zhang, J. Bioorg. Med. Chem. Lett. 2015, 25, 1961.

[67] Kaur, A.; Singh, V. Synlett 2014, 1191.

[68] Liu, X. R.; Chen, C.; Xiu, Y. H.; Chen, A. J.; Guo, L.; Zhang, R.; Chen, J. Z.; Hou, Z. S. Catal. Commun. 2015, 67, 90.

[69] Shaterian, H. R.; Aghakhanizadeh, M. Phosphorus, Sulfur Silicon Relat. Elem. 2013, 188, 1064.

[70] Fang, D.; Zhang, D. Z.; Liu, Zu. L. Monatsh. Chem. 2010, 141, 419.

[71] He, L. Q.; Qin, S. J.; Chang, T.; Sun, Y. Z.; Zhao, J. Q. Int. J. Mol. Sci. 2014, 15, 8656.

[72] Nagarajan, S.; Kandasamy, E. Catal. Lett. 2014, 144, 1507.

[73] Dayanand, P.; Dattatray, C.; Abhijeet, M. Catal. Lett. 2014, 144, 949.

[74] Heravi, M. M.; Saeedi, M.; Karimi, N.; Zakeri, M.; Beheshtiha, Y. S.; Davoodnia, A. Synth. Commun. 2010, 40, 523.

[75] Ying, A. G.; Liu, S.; Yang, J. G.; Hu, H. N. Ind. Eng. Chem. Res. 2014, 53, 16143 .

(Zhao, X.) 